\title{
Computational Prediction of Asparagine and Aspartate Hydroxylation Sites
}

\section{on Human Proteins}

\author{
By \\ Zhen Liu, B.Sc.H. \\ A thesis submitted to \\ the Faculty of Graduate Studies and Research \\ in partial fulfillment of \\ the requirements for the degree of
}

Masters of Science

Biology Department

Ottawa-Carleton Institute of Biology

Carleton University

Ottawa, Ontario

June 2009

(C) Copyright

2009 Zhen Liu 
The undersigned hereby recommend to

the Faculty of Graduate Studies and Research

acceptance of the thesis

\title{
Computational Identification of Hydroxylation Sites
}

By

\author{
Zhen Liu, B.Sc.H. \\ in partial fulfilment of the requirements \\ for the degree of Masters of Science
}

Chair, Department of Biology

Thesis Co-Supervisors

Carleton University 


\section{ABSTRACT}

Humans encounter conditions of low oxygen (hypoxia) under various physiological and pathological conditions. Oxygen-dependent post-translational modifications (PTMs) can affect both the physical and chemical properties of proteins. Thus, these modifications are key elements to the cellular response to hypoxia. Asparagine or aspartate hydroxylation are examples of oxygen-dependent PTMs, and play an important role in cellular oxygen sensing. Experimental approaches to study asparagine or aspartate hydroxylation of proteins are expensive and time-consuming. Therefore, there is a need for computational prediction tools to narrow and rank potential protein targets of oxygen-dependent PTMs for experimental validation. Following a detailed review of the literature, we found 40 experimentally confirmed human protein targets with 61 positive sites for asparagine/aspartate hydroxylation. Utilizing the RVPNET tool to predict solvent accessibility, it was found that almost all positive sites were located on the protein surface. An analysis of predicted protein secondary structure, found that all positive sites occurred in non-regular or beta-strand secondary structures. A sequence logo tool, which displays positional information of aligned amino acid sequences, found that the sequence windows surrounding each positive site showed some pattern of sequence conservation. We used a support vector machine to train a classifier on the positive sites using the input features of surface accessibility, secondary structure, and position specific scoring matrix (PSSM). This trained classifier can recall 92.73\% asparagine/aspartate hydroxylation sites with $61.45 \%$ precision based on leave-one-out test method. The prediction tool - HYPT is currently available at http://bioinf.sce.carleton.ca/HYPT. 


\section{ACKNOWLEDGEMENTS}

First of all, I gratefully acknowledge my two supervisors, Dr. William Willmore from the Biology Department and Dr. James Green from the Department of Systems and Computer Engineering. They provided me consistent support and guidance. Dr. Willmore trained me in the biological background knowledge, which helped me to have a clear objective of my project. He provided as many resources as he could, not only the relevant papers and tools, but also some expertise in bioinformatics field. All these resources benefited me a lot, and helped me to steer towards the right track of my project. Since most parts of my project involved programming, Dr. Green provided me a huge support by supplying server, database and coding. He also provided the source code that I could utilize in my own programming. He helped me to find and install a good tool that I used to train the classifier as well as debugging. I learned many programming skills from him. During my MSc. studies, Drs. Willmore and Green gave me insightful ideas and suggestions with their kindness. Thank you!

I would like to thank Dr. Guy Drouin from University of Ottawa, for being my committee member, and giving me helpful support and comments. I would like to thank my lab mates, who are friendly and make my graduate studies smooth. I would also like to thank Natural Sciences and Engineering Research Council of Canada for partially funding this research.

Finally, I would like to express my appreciation to my family. They love me, believe me, support me and encourage me all these years. Thank you, my dear husband and my loving son. 


\section{TABLE OF CONTENTS}

ABSTRACT iii

ACKNOWLEDGEMENTS

TABLE OF CONTENTS

LIST OF FIGURES AND TABLES viii

LIST OF ABBREVIATIONS - xi

1. INTRODUCTION 1

1.1. Hypoxia and PTM 1

1.2. Hydroxylation as a PTM 4

1.3. Existing computational tools for prediction PTM sites 9

$\begin{array}{ll}\text { 1.4. Hypothesis } & 10\end{array}$

1.5. Overview of research results 11

2. MATERIALS AND METHODS 12

2.1. Outline 12

2.2. Data collection and storage 12

2.2.1. Positive and negative data sets 12

2.2.2. Local database 19

$\begin{array}{ll}\text { 2.3. Characterizing target proteins } & 20\end{array}$

$\begin{array}{ll}\text { 2.3.1. Surface accessibility } 20 & 20\end{array}$

2.3.2. Secondary structure 23 
2.3.3. Patterns of sequence conservation

2.4. Pattern classification

2.4.1. Local sequence window

2.4.2. Removing identical data points

2.4.3. Support vector machines

2.4.4. Analysis of different aspects of the SVM

2.4.5. Evaluation of classification accuracy

2.5. Developing a web server to predict novel hydroxylation sites

3. RESULTS

3.1. Characterization of known asparagine/aspartate hydroxylation sites

3.1.1. Surface accessibility information on the positive sites

3.1.2. Secondary structure information on the positive sites

3.1.3. Sequence conservation information on the positive sites

3.2. Pattern classification

3.2.1. The effect of class imbalance on the SVM classifier

3.2.2. The effect of feature selection on the SVM classifier

3.2.3. Using differential positive/negative misclassification cost ratios to address class imbalance

3.2.4. The effect of domains on the SVM classifier 70

3.2.5. The optimal and final classifiers 73

3.3 The hydroxylation prediction web server 75 
4.1 Summary of contributions $\quad 80$

4.1.1. Data collection and storage $\quad 80$

4.1.2. Characterizing target proteins 81

$\begin{array}{ll}\text { 4.1.3. Pattern classification } & 81\end{array}$

4.1.4. The hydroxylation prediction web server 82

4.2 Potential improvement $\quad 82$

$\begin{array}{ll}\text { 4.2.1. Training dataset } & 82\end{array}$

4.2.2. Compute different feature data 85

4.2.3. Effect of domain 86

4.2.4. Size of local sequence window 86

4.2.5. Train classifiers for asparagine and aspartate hydroxylation $\begin{array}{ll}\text { separately } & 87\end{array}$

4.3. Directions for future work 94

4.3.1. Application of tool to all human proteins 94

4.3.2. Application of studying model to other types of hydroxylation 94

REFERENCES 96 


\section{LIST OF FIGURES AND TABLES}

Figure 1: The general process from gene to PTM

Table 1: The seven families of hydroxylases

Figure 2: The concept of mass spectrometry

Figure 3: The steps taken for developing the hydroxylation prediction tool

Table 2: The experimentally verified asparagine/aspartate hydroxylation sites for Homo sapiens

Figure 4: A portion of the table that contains the surface accessibility information

Figure 5: A portion of the table that contains the secondary structure information

Figure 6: A portion of the table that contains the PSSM information

Figure 7: Basic concepts about support vector machine

Figure 8: Kernel methods can deal with nonlinear classifier

Table 3: The protein targets that contain EGF domain or ankyrin repeat domain

Figure 9: Confusion matrix

Table 4: The predicted surface accessibility of 61 positive sites

Table 5: The predicted secondary structure of 61 positive sites

Figure 10: PDB structure of HIF- $\alpha$ being hydroxylated by the Factor Inhibiting HIF asparagine hydroxylase

Figure 11: The conservation pattern of sequence windows of size 15 for the 61 positive sites

Figure 12: The effect of positive/negative ratio to the classifier trained with 3 features of PSSM, surface accessibility and secondary structure 
Figure 13: The effect of positive/negative ratio to the classifier trained with surface accessibility feature only

Figure 14: The effect of positive/negative ratio to the classifier trained with secondary structure feature only

Figure 15: The effect of positive/negative ratio to the classifier trained with PSSM feature only

Figure 16: The effect of positive/negative ratio to the classifier trained with surface accessibility and secondary structure features

Figure 17: The effect of positive/negative ratio to the classifier trained with PSSM and surface accessibility

Figure 18: The effect of positive/negative ratio to the classifier trained with PSSM and secondary structure

Table 6: The accuracy of the classifiers with different positive/negative weight cost

Table 7: The accuracy of trained classifier based on EGF domain information

Table 8: The accuracy of trained classifier based on ankyrin domain information

Table 9: Comparison of different trained classifiers

Figure 19: The screen shot of the interface of the prediction web service

Figure 20: The screen shot of part of the web page that returns the predicted results

Figure 21: The effect of different training set size to the accuracy of trained SVM classifier by using the leave-one-out method 
Figure 22: The conservation pattern of sequence windows of size 17 for the 61 positive sites

Figure 23: The conservation pattern of sequence windows for the 44 positive $\mathrm{N}$ sites

Figure 24: The conservation pattern of sequence windows for the 17 positive D sites 


\section{LIST OF ABBREVIATIONS}

EC: Enzyme Commission Number

EGF: Epidermal Growth Factor

ELM: Eukaryotic Linear Motif

FN: False Negatives

FP: False Positives

MCC: Matthew's Correlation Coefficient

NCBI: National Center for Biotechnology Information

PCI-SS: Parallel Cascade Identification based Protein Secondary Structure Prediction Server

PPV: Positive Predictive Value

PSI-BLAST: Position Specific Iterative BLAST

PSSM: Position Specific Scoring Matrix

PTM: Post-translational modification

RBF: Radial Basis Function

SOAP: Simple Object Access Protocol

SVM: Support Vector Machine

THB: Tetrahydrobiopterin

TN: True Negatives

TP: True Positives 


\section{INTRODUCTION}

\subsection{Hypoxia and PTM}

As humans, we cannot live without oxygen. However, humans encounter conditions of low oxygen (hypoxia) under various physiological and pathological conditions, such as high altitude and ischemic disease. For example, stroke (the fourth leading cause of death in Canada) is caused by blockage of blood flow to brain. Cardiac arrest is caused by the abrupt cessation of normal circulation of the blood to a portion of the heart. Anemia, sleep apnea and most respiratory diseases are caused by defects in oxygen transportation. All are examples of severe hypoxic situations. The human response to hypoxia is to initiate both gene and protein expression in order to return the cell to homeostasis or normoxic function. This is controlled by alteration in protein function that is dependent upon the levels of ambient oxygen. In many cases, this requires the addition of one or both oxygen atoms in dimolecular oxygen to be added to proteins. Oxygen-dependent post-translational modifications (PTMs) can affect both physical and chemical properties of proteins and thus are the key to the cellular response to hypoxia.

PTMs are chemical modifications of proteins after their translation. As shown in Figure 1 (Jensen, 2008), genomic DNA is used to synthesize RNA during transcription. The initial transcript then moves from the nucleus to the cytosol, where the introns are spliced out and the exons are spliced together to form the mature transcript (at the spliceosome). This is where alternative splicing (of exons) may occur. Protein is then 
Figure 1. The general process from gene to PTM (reproduced from Jensen (2008)). Genomic DNA is used to synthesize RNA during transcription. After alternative splicing, the exons are joined together to form mRNA. Protein is then synthesized from mRNA with the process of translation. The final protein can then be modified with PTM process. Phosphorylation, ubiquitination, lipid anchor, and glycosylation are some typical types of PTMs. 


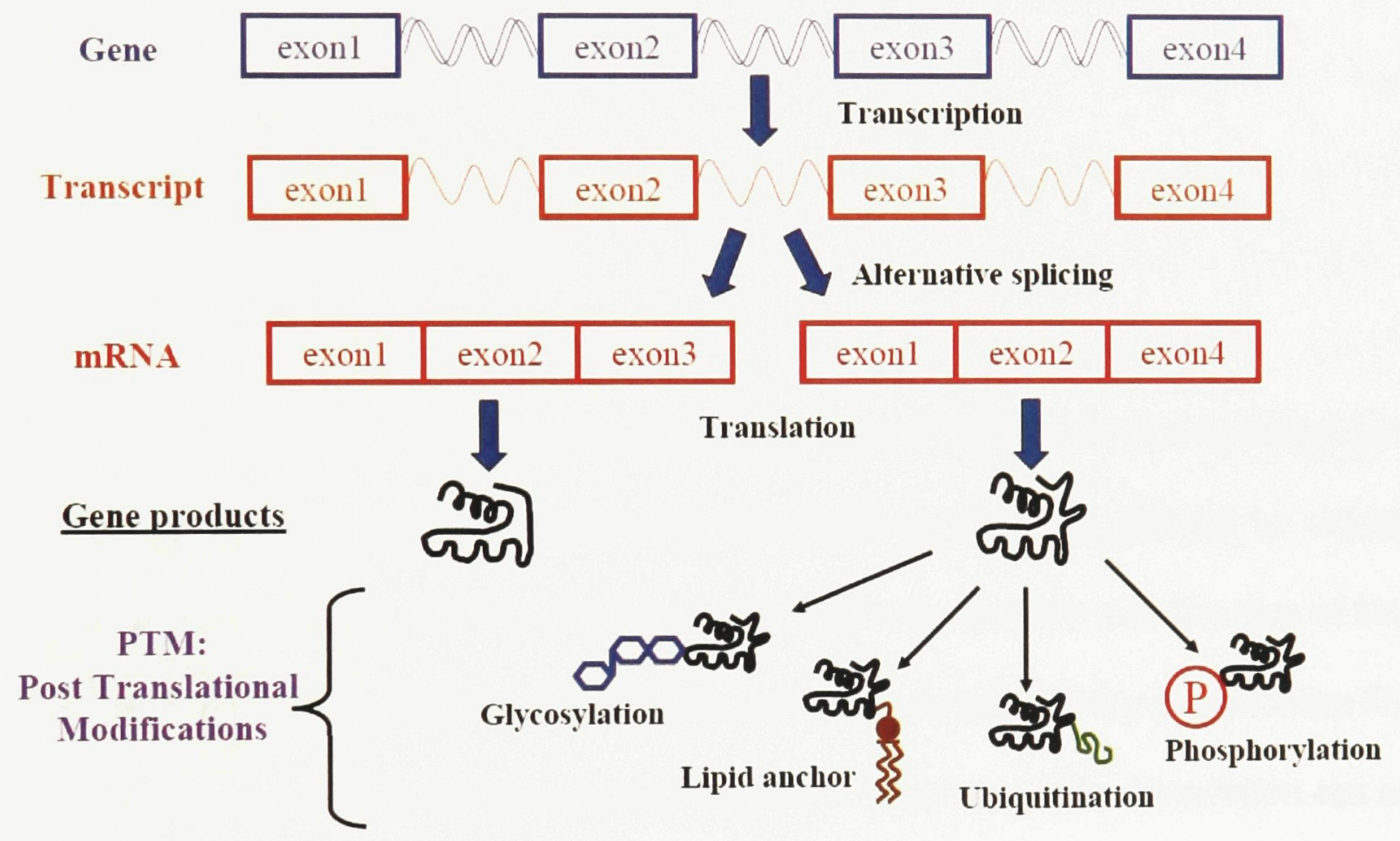


synthesized from mRNA in the process of translation. The final protein can then be modified by a number of PTMs, once it is made. For example, phosphorylation is one type of PTM in which a phosphate group is added to a protein. Ubiquitination involves the covalent attachment of ubiquitin monomers to the protein, usually signalling for its degradation. Glycosylation is the addition of polysaccharides to a protein, and is important for protein folding. PTMs not only change the structure of the proteins, but also alter the function of the proteins, and therefore provide an important mechanism of control over cellular state.

\subsection{Hydroxylation as a PTM}

Hydroxylation is one type of oxygen-dependent PTM, in which a hydroxyl group $(-\mathrm{OH})$ is added to a protein's amino acid side chain; a reaction catalyzed by enzymes known as hydroxylases. Hydroxylation not only alters the structure and function of target proteins, but may also facilitate other PTMs. Since hydroxylation only occurs when $\mathrm{O}_{2}$ is present, hypoxia inhibits the hydroxylation process. Inhibition of hydroxylation can also mimic the hypoxic environment, initiating cellular defense mechanisms for the survival of severe hypoxic conditions. Proteins that can be hydroxylated are therefore potential determining points for cellular hypoxia survival and the minimization of damage resulting from the hypoxic condition.

Many hydroxylases hydroxylate chemical compounds in the presence of oxygen.

These include the monoxygenase family of hydroxylases. With the exception of phenylalanine hydroxylase, tyrosine hydroxylase and tryptophan hydroxylase, only 
certain hydroxylases of the dioxygenase family of enzymes can utilize amino acids as substrates, including those incorporated into proteins.

Table 1 show the four families of dioxygenase and three families of monoxygenases respectively. Dioxygenases catalyze the reaction below:

\section{Amino acid + 2-oxoglutarate $+\mathrm{O}_{2} \leftrightarrow$ hydroxy-amino acid + succinate $+\mathrm{CO}_{2}$}

Monoxygenases catalyze the reaction below $(\mathrm{THB}=$ tetrahydrobiopterin):

\section{Amino acid + THB $+\mathrm{O}_{2} \leftrightarrow$ hydroxyl-amino acid + hydroxyl-THB}

The dioxygenase families of hydroxylases modify amino acids present in proteins involved in control of cellular function (transcription factors), cellular signalling (receptors), cellular transport (ion channels), etc. The dioxygenase families of hydroxylases include proline hydroxylases, asparagine hydroxylases, aspartate hydroxylases, and lysine hydroxylases. My research focused on asparagine and aspartate hydroxylases, because there were more experimentally validated targets of these hydroxylases.

Mass spectrometry is the only experimental approach to identify hydroxylation events on proteins. Mass spectrometry generates gas-phase ions from a sample, separates them according to their mass-to-charge ratio $(\mathrm{m} / \mathrm{z})$ and determines their abundance. As shown in Figure 2, a PTM can be identified by comparing the spectra of modified and non-modified proteins (Jensen, 2008). For hydroxylation, this involves comparing the spectra of hydroxylated and non-hydroxylated proteins. There is a $+16 \mathrm{~m} / \mathrm{z}$ shift from nonhydroxylated to hydroxylated peptides, the molecular mass of one oxygen atom. To date, only a small collection of target proteins, hydroxylated by dioxygenases, have been 

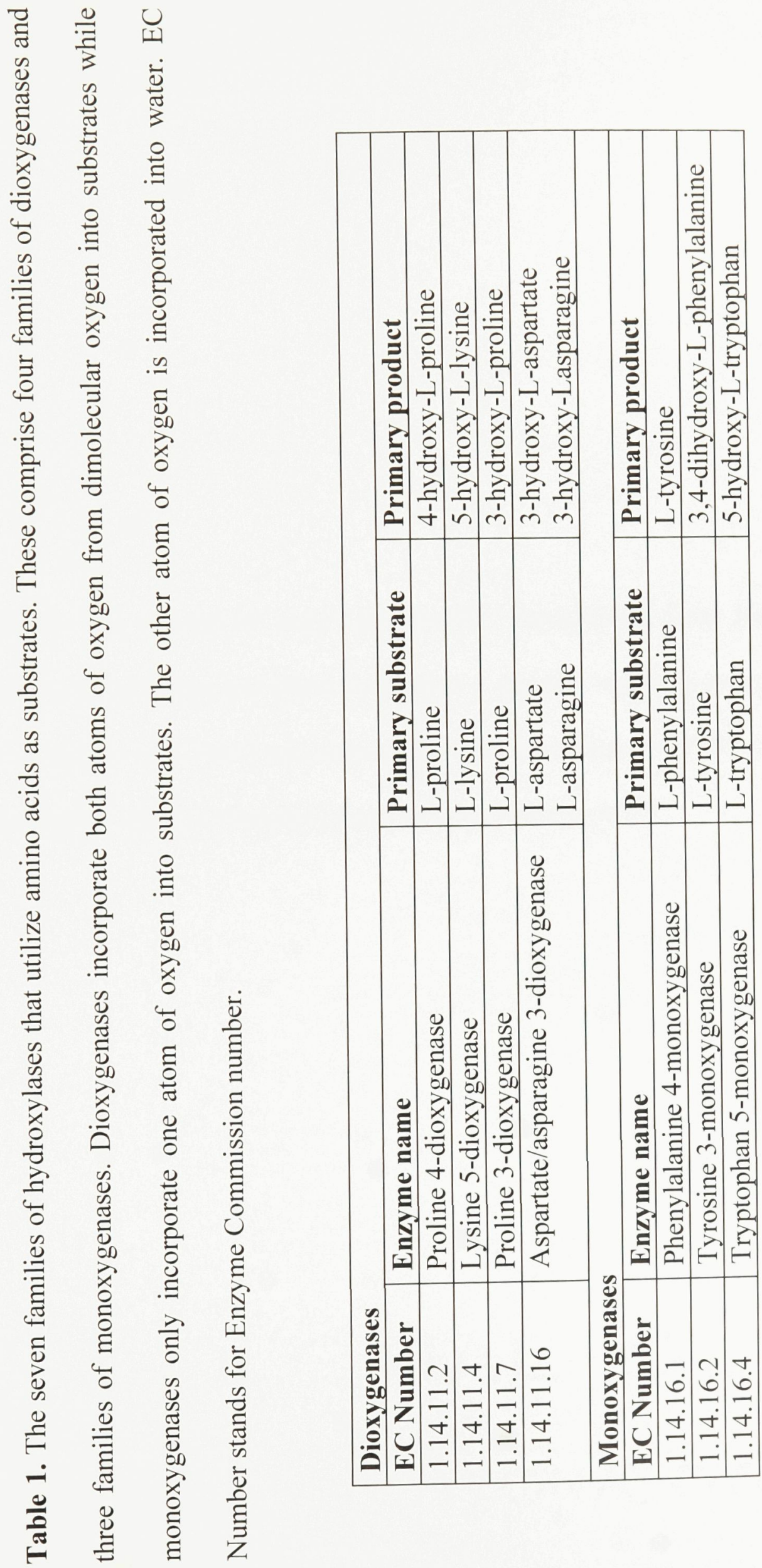
Figure 2. The concept of mass spectrometry (reproduced from Jensen, 2008). Mass spectrometry generates gas-phase ions from a sample, separates them according to their mass-to-charge ratio $(\mathrm{m} / \mathrm{z})$ and determines their abundance. A PTM can be identified by comparing the spectra of modified and non-modified proteins. 


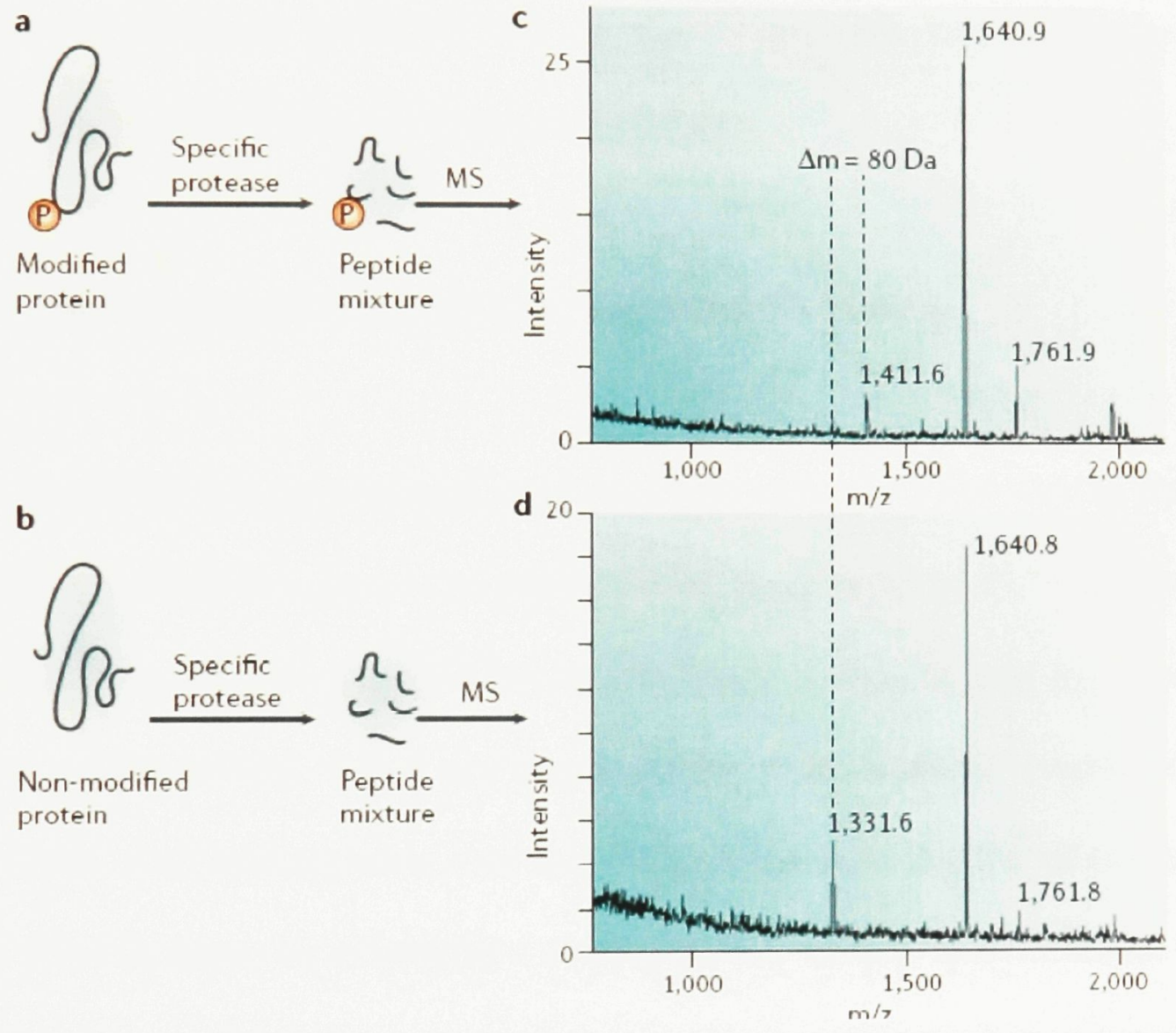


identified experimentally (Stenflo et al., 1987; Przysiecki et al., 1987; Coleman et al., 2007). For example, only 11 human protein targets of asparagine hydroxylation are currently listed in dbPTM. Experimental approaches are expensive and time-consuming and only the protein targets that have a very high potential to be modified in an oxygendependent manner should be considered for experimental validation. Therefore there is a need for computational prediction tools to narrow and rank lists of protein targets of oxygen-dependent PTMs for experimental validation.

\subsection{Existing computational tools for predicting PTM sites}

A number of computational tools exist for predicting PTM sites in proteins. For example, AutoMotif is a server tool for predicting many types of PTMs (Plewczynski et al., 2005). However, it is not available for asparagine or aspartic acid hydroxylation and can only predict proline, lysine and phenylalanine hydroxylation. Overall, it claims to recall $70.8 \%$ of all hydroxylation sites with $69.27 \%$ precision. AutoMotif uses an SVM to predict PTM locations by examining a local sequence window of 9-13 amino acids. The positive training examples are drawn from annotated sequences from Swiss-Prot while the negative training examples are taken from Swiss-Prot sequences that have not annotated PTM. The training sequences are encoded using a variety of techniques including BLOSUM, sequence profile from a multiple sequence alignment, or a simple binary orthogonal encoding (twenty 0/1 values for each amino acid) (Plewczynski et al., 2005). When predicting PTM locations on a protein, the AutoMotif server first checks the 
local sequence window for an exact match against the positive training examples, then runs the SVM.

The Eukaryotic Linear Motif Resource (ELM) for Functional Sites in Proteins can identify functional sites based on known linear motifs which capture local sequence conservation. However, it can only identify asparagine/aspartate hydroxylation sites that are within epidermal growth factor (EGF) domain containing proteins. Many experimentally verified asparagine/aspartate hydroxylation sites that occur outside of EGF domain-containing proteins, cannot be identified by ELM. Furthermore, many asparagines/aspartates within EGF domains may not be hydroxylated. Currently, we have not found any prediction tool that is specific for hydroxylation of asparagine or aspartate. Thus, we are the first group to develop a tool for the identification of asparagine or aspartate hydroxylation sites on proteins and to rank candidate proteins for experimental validation of hydroxylation events.

\subsection{Hypothesis}

We hypothesize that asparagine/aspartate hydroxylation sites on proteins have common features. We predict that the known hydroxylation sites will a) have similar local sequence conservation patterns, b) be found at the surface of the proteins and c) be located in similar secondary structure of proteins. Furthermore, we hypothesize that support vector machines can effectively predict hydroxylation sites based solely on protein sequence, secondary structure, and surface accessibility. 


\subsection{Overview of research results}

My research includes four main stages.

Stage 1 is to collect all protein targets that are experimentally confirmed for asparagine/aspartate hydroxylation. Through a detailed literature review, we found 40 protein targets with 61 positive sites for asparagine/aspartate hydroxylation.

Stage 2 is to analyze the positive sites in terms of sequence conservation, secondary structure, and solvent accessibility. We aligned the local sequence windows ( \pm 7 amino acids centered at each positive site), and found strong sequence conservation patterns. Also, we predicted the surface accessibility and secondary structure for these protein targets and found that, with the exception of one site, all positive sites were located on the protein surface, and all sites were located in non-regular or beta-strand secondary structure elements. Therefore, we predict hydroxylation sites not only based on sequence information, but also based on sequence-derived information.

Stage 3 is to develop and evaluate a prediction tool. A support vector machine (SVM) was used to train a classifier with feature inputs of surface accessibility, secondary structure, and position specific scoring matrix (PSSM). This trained classifier can recall $92.73 \%$ of asparagine/aspartate hydroxylation sites with $61.45 \%$ precision based on leave-one-out test method.

Stage 4 is to develop a web server to make this prediction tool available to the research community. The prediction tool - HYPT is currently available at http://bioinf.sce.carleton.ca/HYPT. 


\section{MATERIALS AND METHODS}

\subsection{Outline}

The basic steps of developing the prediction tool are illustrated in Figure 3. Step 1 is to find the protein targets and get the training dataset. Step 2 is to compute three features for each protein targets: surface accessibility, secondary structure and local sequence conservation information. Step 3 is to train an SVM to recognize and predict hydroxylation sites based on the information contained in the three features. Step 4 is to evaluate classification accuracy and get the best classifier possible. Step 5 is to predict and rank asparagine/aspartate hydroxylation protein targets on all human proteins. Lastly, the SVM-based prediction method has been implemented as a web server to make it available to the research community.

\subsection{Data collection and storage}

\subsubsection{Positive and negative data sets}

In order to narrow the scope of proteins that could be used, we focused solely on human proteins. Proteins from other organisms will eventually be utilized, but for training the SVM, only human proteins were utilized for the present study. We utilized dbPTM and an in-depth review of the literature to identify the protein targets with known 
Figure 3. The basic steps of developing an asparagine/aspartate hydroxylation prediction tool. Step 1 is to find the asparagine/aspartate hydroxylated protein targets that are experimentally validated, and get the training dataset. Step 2 is to compute three features of the protein targets: surface accessibility, secondary structure and conservation pattern information. Step 3 is to use a support vector machine to train the information of the three features. Step 4 is to analyze and get the best classifier. Step 5 is to predict and rank asparagine/aspartate hydroxylation protein targets. 


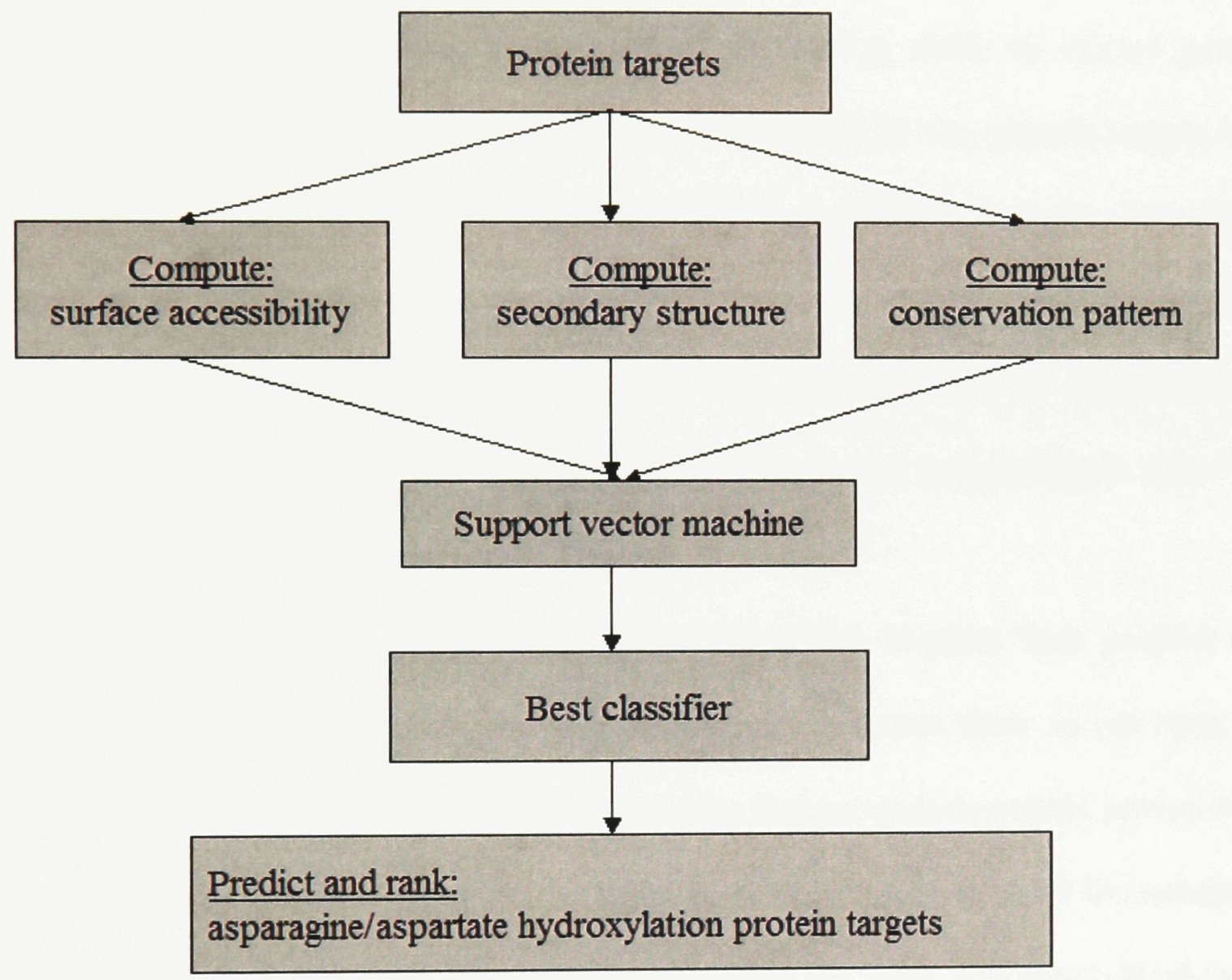


hydroxylation sites. dbPTM is a database that provides experimentally verified PTMs. It includes all of the experimentally validated PTM sites from Swiss-Prot, PhosphoELM and O-GLYCBASE (Lee et al., 2006). dbPTM listed 19 protein targets with 21 asparagine/aspatate hydroxylation sites. An in-depth literature review was conducted which took significant effort. I searched Pubmed, Brenda, Enzyme, and Swiss-Prot using keywords "asparagine, aspartate, hydroxylation" to find as many as related journal articles as possible. After reviewing all the articles, I found 21 new protein targets with 40 asparagine/aspartate hydroxylation sites (Stenflo et al., 1987; Przysiecki et al., 1987; Coleman et al., 2007) that were not previously listed in dbPTM. This represents a doubling of the previously documented targets. In total, we found 40 experimentally confirmed human protein targets with 61 asparagine/aspartate hydroxylation sites. The positive site information is presented in Table 2.

In general, a learning algorithm, such as an SVM, requires both positive and negative data to build a classifier that will discriminate between them. In our case, the positive data are all the asparagine/aspartate residues that are experimentally proven to be hydroxylated on target proteins, while the negative sites are taken to be all the remaining (i.e. non-validated) asparagine/aspartate residues within the same 40 hydroxylated target proteins. This resulted in 1,980 non-hydroxylated sites in total.

While we cannot be certain that all non-validated amino acids are, in fact, true negative sites, we were forced to use this definition due to a paucity of documented negative data in the literature. Many existing and successful PTM prediction tools define 
Table 2. The experimentally verified asparagine/aspartate hydroxylation sites for Homo sapiens. Column 'ref_num' shows the reference sequence number of the protein. Column Protein name shows the name of the protein. Column 'Hydrox. Residue' shows the hydroxylated amino acid, either asparagine (N) or aspartate (D). Column 'Pos.' gives the position of the amino acid within the protein sequence. Column 'Resource' shows the resource that the validated information comes from. This column either indicates that this site is documented in dbPTM, or the PMID number of the relevant paper is given. Note that dbPTM is a database that provides experimentally verified PTMs.

\begin{tabular}{|c|c|c|c|c|}
\hline Ref_num & Protein name & $\begin{array}{l}\text { Hydrox. } \\
\text { Residue }\end{array}$ & Pos. & Resource \\
\hline NP_001521.1 & $\begin{array}{l}\text { hypoxia-inducible factor } 1 \text {, alpha } \\
\text { subunit isoform } 1\end{array}$ & $\mathrm{~N}$ & 803 & dbPTM \\
\hline NP 003989.2 & nuclear factor kappa-B, subunit 1 & $\mathrm{~N}$ & 679 & 17003112 \\
\hline \multirow[t]{2}{*}{ NP_065390.1 } & \multirow{2}{*}{$\begin{array}{l}\text { nuclear factor of kappa light } \\
\text { polypeptide gene enhancer in B-cells } \\
\text { inhibitor, alpha }\end{array}$} & $\mathrm{N}$ & 210 & 17003112 \\
\hline & & $\mathrm{N}$ & 244 & 17003112 \\
\hline NP 000124.1 & coagulation factor IX & $\mathrm{D}$ & 110 & 2726737 \\
\hline NP 000495.1 & coagulation factor $\mathrm{X}$ preproprotein & $\mathrm{D}$ & 103 & dbPTM \\
\hline NP_000122.1 & $\begin{array}{l}\text { coagulation factor VII precursor, } \\
\text { isoform a }\end{array}$ & $\mathrm{D}$ & 123 & dbPTM \\
\hline NP_062562.1 & $\begin{array}{l}\text { coagulation factor VII precursor, } \\
\text { isoform } b\end{array}$ & $\mathrm{D}$ & 101 & 2726737 \\
\hline \multirow[t]{4}{*}{ NP_000304.1 } & \multirow[t]{4}{*}{ protein S (alpha) } & $\mathrm{D}$ & 136 & dbPTM \\
\hline & & $\mathrm{N}$ & 177 & 2948188 \\
\hline & & $\mathrm{N}$ & 219 & 2948188 \\
\hline & & $\mathrm{N}$ & 258 & 2948188 \\
\hline NP_003882.1 & $\begin{array}{l}\text { protein } \mathrm{Z} \text {, vitamin } \mathrm{K} \text {-dependent plasma } \\
\text { glycoprotein }\end{array}$ & $\mathrm{D}$ & 104 & dbPTM \\
\hline NP_001724.3 & $\begin{array}{l}\text { complement component } 1, \mathrm{r} \\
\text { subcomponent }\end{array}$ & $\mathrm{N}$ & 167 & dbPTM \\
\hline NP_958850.1 & $\begin{array}{l}\text { complement component } 1, \mathrm{~s} \\
\text { subcomponent }\end{array}$ & $\mathrm{N}$ & 149 & dbPTM \\
\hline NP_001725.1 & $\begin{array}{l}\text { complement component } 1, \mathrm{~s} \\
\text { subcomponent }\end{array}$ & $\mathrm{N}$ & 149 & 2825166 \\
\hline \multirow[t]{2}{*}{ NP_003352.2 } & \multirow[t]{2}{*}{ uromodulin precursor } & $\mathrm{N}$ & 85 & 2825166 \\
\hline & & $\mathrm{N}$ & 128 & 2825166 \\
\hline
\end{tabular}




\begin{tabular}{|c|c|c|c|c|}
\hline \multirow{2}{*}{$\begin{array}{l}\text { NP_0010083 } \\
90.1\end{array}$} & \multirow[t]{2}{*}{ uromodulin precursor } & $\mathrm{N}$ & 85 & 2825166 \\
\hline & & $\mathrm{N}$ & 128 & 2825166 \\
\hline \multirow[t]{2}{*}{ NP_060087.2 } & \multirow[t]{2}{*}{ notch1 preproprotein } & $\mathrm{N}$ & 1956 & 17573339 \\
\hline & & $\mathrm{N}$ & 2023 & 17573339 \\
\hline \multirow[t]{2}{*}{ NP_077719.2 } & \multirow[t]{2}{*}{ notch 2 preproprotein } & $\mathrm{N}$ & 1904 & 17573339 \\
\hline & & $\mathrm{N}$ & 1971 & 17573339 \\
\hline \multirow[t]{2}{*}{ NP_000426.1 } & \multirow[t]{2}{*}{ Notch homolog 3} & $\mathrm{~N}$ & 1866 & 17573339 \\
\hline & & $\mathrm{N}$ & 1933 & 17573339 \\
\hline NP_000303.1 & $\begin{array}{l}\text { protein } \mathrm{C} \text { (inactivator of coagulation } \\
\text { factors } \mathrm{Va} \text { and VIIIa) }\end{array}$ & $\mathrm{D}$ & 113 & dbPTM \\
\hline \multirow[t]{8}{*}{ NP_000205.1 } & \multirow[t]{8}{*}{ jagged 1 precursor } & $\mathrm{N}$ & 504 & 11773073 \\
\hline & & $\mathrm{N}$ & 542 & 11773073 \\
\hline & & $\mathrm{D}$ & 391 & 11773073 \\
\hline & & $\mathrm{D}$ & 466 & 11773073 \\
\hline & & $\mathrm{D}$ & 646 & 11773073 \\
\hline & & $\mathrm{D}$ & 684 & 11773073 \\
\hline & & $\mathrm{D}$ & 799 & 11773073 \\
\hline & & $\mathrm{D}$ & 837 & 11773073 \\
\hline \multirow[t]{2}{*}{ NP_002499.1 } & \multirow[t]{2}{*}{ nidogen (enactin) } & $\mathrm{N}$ & 729 & 2496973 \\
\hline & & $\mathrm{N}$ & 819 & 2496973 \\
\hline NP 056137.1 & fem-1 homolog $b$ & $\mathrm{~N}$ & 526 & 17003112 \\
\hline NP_002032.2 & $\begin{array}{l}\text { GA binding protein transcription factor, } \\
\text { beta subunit } 2 \text { isoform gamma } 1\end{array}$ & $\mathrm{~N}$ & 98 & 17003112 \\
\hline NP_005245.2 & $\begin{array}{l}\text { GA binding protein transcription factor, } \\
\text { beta subunit } 2 \text { isoform beta } 1\end{array}$ & $\mathrm{~N}$ & 98 & 17003112 \\
\hline NP_057738.1 & $\begin{array}{l}\text { GA binding protein transcription factor, } \\
\text { beta subunit } 2 \text { isoform beta } 2\end{array}$ & $\mathrm{~N}$ & 98 & 17003112 \\
\hline NP_057739.1 & $\begin{array}{l}\text { GA binding protein transcription factor, } \\
\text { beta subunit } 2 \text { isoform gamma } 2\end{array}$ & $\mathrm{~N}$ & 98 & 17003112 \\
\hline NP_852092.1 & $\begin{array}{l}\text { GA binding protein transcription factor, } \\
\text { beta subunit } 2 \text { isoform gamma } 2\end{array}$ & $\mathrm{~N}$ & 98 & 17003112 \\
\hline NP_003738.2 & $\begin{array}{l}\text { tankyrase, TRF1-interacting ankyrin- } \\
\text { related ADP-ribose polymerase }\end{array}$ & $\mathrm{N}$ & 864 & 17003112 \\
\hline NP_002805.1 & $\begin{array}{l}\text { proteasome } 26 \mathrm{~S} \text { non-ATPase subunit } 10 \\
\text { isoform } 1\end{array}$ & $\mathrm{~N}$ & 100 & 17003112 \\
\hline NP_002471.1 & $\begin{array}{l}\text { protein phosphatase } 1 \text {, regulatory } \\
\text { (inhibitor) subunit } 12 \mathrm{~A}\end{array}$ & $\mathrm{~N}$ & 226 & 17003112 \\
\hline NP 665807.1 & myotrophin & $\mathrm{N}$ & 62 & 17003112 \\
\hline NP 060174.2 & fetal globin inducing factor & $\mathrm{N}$ & 168 & 17003112 \\
\hline \multirow[t]{2}{*}{ NP_000618.2 } & \multirow{2}{*}{$\begin{array}{l}\text { latent transforming growth factor beta } \\
\text { binding protein } 1 \text { isoform LTBP-1S }\end{array}$} & $\mathrm{N}$ & 649 & dbPTM \\
\hline & & $\mathrm{N}$ & 812 & dbPTM \\
\hline NP_000352.1 & thrombomodulin precursor & $\mathrm{N}$ & 342 & dbPTM \\
\hline \multirow[t]{2}{*}{ NP_055061.1 } & \multirow{2}{*}{$\begin{array}{l}\text { Cadherin EGF LAG seven-pass G-type } \\
\text { receptor } 1\end{array}$} & $\mathrm{~N}$ & 1666 & dbPTM \\
\hline & & $\mathrm{D}$ & 1889 & dbPTM \\
\hline NP_ 001399.1 & Cadherin EGF LAG seven-pass G-type & $\mathrm{N}$ & 1591 & dbPTM \\
\hline
\end{tabular}




\begin{tabular}{|l|l|c|c|c|}
\hline & receptor 2 & $\mathrm{N}$ & 1810 & dbPTM \\
\hline NP_001421.2 & $\begin{array}{l}\text { Endothelial PAS domain-containing } \\
\text { protein 1 }\end{array}$ & $\mathrm{N}$ & 847 & dbPTM \\
\hline Q14766 & $\begin{array}{l}\text { Latent-transforming growth factor beta- } \\
\text { binding protein, isoform 1L }\end{array}$ & $\mathrm{N}$ & 848 & dbPTM \\
\cline { 2 - 5 } & N & 1011 & dbPTM \\
\hline NP_006601.2 & Mannan-binding lectin serine protease 2 & $\mathrm{N}$ & 158 & dbPTM \\
\hline P00740 & $\begin{array}{l}\text { Cadherin EGF LAG seven-pass G-type } \\
\text { receptor 3 }\end{array}$ & $\mathrm{D}$ & 1963 & dbPTM \\
\hline
\end{tabular}


negative sites as we have done here (Plewczynski et al., 2005; Julenius et al., 2004). For example, hydroxylation prediction in AutoMotif was trained on 363 positive points and 10,000 negative points, can achieve $70.80 \%$ sensitivity and $69.27 \%$ precision (Plewczynski et al., 2005). Acetylation prediction in AutoMotif, trained on 552 positive points and 10,014 negative points, can achieve $89.86 \%$ sensitivity and $95.75 \%$ precision (Plewczynski et al., 2005). The sumoylation prediction tool trained on 62 positive sites and 1,560 negative sites, can achieve $94 \%$ sensitivity and $17 \%$ specificity (Green et al., 2006). These examples gave us support for the use of our negative dataset as defined above.

\subsubsection{Local database}

We need a local database to store all the information for analysis. MySQL was used to create a local database. Programs were written in PHP and PERL to compute and parse all necessary information into this database. I created a table to store all human protein sequence data. These data were parsed from the file protein.fa, which was downloaded from the National Center for Biotechnology Information (NCBI) at $\mathrm{ftp} / / / \mathrm{ftp} . n c b i . n i h . g o v / r e f s e q / H \_s a p i e n s / H \_s a p i e n s / p r o t e i n /$ in October of 2008, and contains the amino acid sequence of all 34,180 human proteins. A table was created to store all the positive and negative site locations on all identified protein targets. As discussed below, a number of tables were also created to store the information for surface accessibility, secondary structure, and sequence conservation. 


\subsection{Characterizing target proteins}

In this study, each target protein is characterized by its surface accessibility, secondary structure, and pattern of sequence conservation. These three features are used to compare all 40 identified target proteins and then used as input data to train an SVM hydroxylation prediction tool. Each feature is described below.

\subsubsection{Surface accessibility}

RVP-NET is a computational tool for the prediction of protein surface accessibility. It is based on a neural network model which predicts real valued solvent accessibility (i.e. not binary 'buried' vs. 'exposed') from sequence information. It can predict each residue in a protein sequence to be buried or exposed with an accuracy of 19.5\% prediction error (Ahmad et al., 2003). The RVP-NET software is freely available, and was downloaded from http://gibk26.bse.kyutech.ac.jp/jouhou/shandar/netasa/rvp-net/. I ran the tool locally to obtain the surface accessibility of each residue of each protein target, and stored this information in the local database created for this study. Figure 4 shows part of the database table that contains the surface accessibility information in the local database. The column SA_percentage represents the surface (or solvent) accessibility value. 
Figure 4. A portion of the database table that contains the surface accessibility information for each residue of each protein sequence. 'SA_percentage' represents a realvalued estimate of the surface (or solvent) accessibility of each amino acid. The 'Category' column gives a simplified binary estimate of surface accessibility as follows: E stands for exposed and B stands for buried. Note that this column was not used in the final design of the SVM prediction tool since the SA_percentage value provides more information. 


$\begin{array}{cllll}\text { ref_num } & \text { res_position } & \text { res } & \text { SA_percentage } & \text { Category } \\ \text { NP_000303.1 } & 1 & \text { M } & 4.67 & \text { B } \\ \text { NP_000303.1 } & 2 & \text { W } & 11.24 & \text { B } \\ \text { NP_000303.1 } & 3 & \text { Q } & 33.30 & \text { E } \\ \text { NP_000303.1 } & 4 & \text { L } & 1.76 & \text { B } \\ \text { NP_000303.1 } & 5 & \text { T } & 11.39 & \text { B } \\ \text { NP_000303.1 } & 6 & \text { S } & 26.13 & \text { E } \\ \text { NP_000303.1 } & 7 & \text { L } & 3.98 & \text { B } \\ \text { NP_000303.1 } & 8 & \text { L } & 3.32 & \text { B } \\ \text { NP_000303.1 } & 9 & \text { L } & 3.83 & \text { B } \\ \text { NP_000303.1 } & 10 & \text { F } & 3.38 & \text { B } \\ \text { NP_000303.1 } & 11 & \text { V } & 4.71 & \text { B } \\ \text { NP_000303.1 } & 12 & \text { A } & 7.12 & \text { B } \\ \text { NP_000303.1 } & 13 & \text { T } & 20.94 & \text { E } \\ \text { NP_000303.1 } & 14 & \text { W } & 7.74 & \text { B } \\ \text { NP_000303.1 } & 15 & \text { G } & 10.38 & \text { B } \\ \text { NP_000303.1 } & 16 & \text { I } & 2.30 & \text { B }\end{array}$




\subsubsection{Secondary structure}

PCI-SS is a parallel cascade identification (PCI)-based protein secondary structure prediction server. It can distinguish between $\alpha$-helices, $\beta$-strands and nonregular structural elements from primary sequence data (Green et al., 2009). Prediction accuracy of PCI-SS is approximately 78\% (Green et al., 2009) making it one of the most accurate methods currently available. Furthermore, a Simple Object Access Protocol (SOAP) interface is provided which simplifies remote invocation of the PCI-SS prediction tool in a multi-platform distributed computational environment (Green et al., 2009).

In the current study, I developed a custom SOAP client to remotely interface with the PCI-SS server to predict protein secondary structure of each target protein and the resulting data was stored into a local MySQL database. Figure 5 shows part of the table that contains the secondary structure information in the local database. Rather than simply outputting the most likely structural class for each amino acid, PCI-SS outputs three real-valued 'distances'. Each unitless distance denotes how unlikely that particular structural class is: EDistance for extended $\beta$-strand, TDistance for non-regular secondary structure (also referred to as "other"), and HDistance for $\alpha$-helix. The most likely structural classification for each amino acid is determined by selecting the class that has the shortest distance. For example, in Figure 5, the first residue is located in the "other" region, because it has shortest distance from non-regular region, which is 0.01 . When training the SVM to recognize hydroxylation sites, all three PCI-SS distances are provided to the SVM as feature data for each amino acid in the local sequence window. 
Figure 5. A portion of the database table that contains the secondary structure information for each residue of each protein sequence. 'Edistance', 'Tdistance', and 'HDistance' reflect the (unitless) dissimilarity between the residue and the three secondary structure classes of extended beta-strand, non-regular secondary structure (we call it "other"), and helix respectively. The residue will be located in the structure region that has the shortest distance. For example, the first residue locates in other region, because it has shortest distance from non-regular region, which is 0.01 . 


$\begin{array}{cllllll}\text { ref_num } & \text { res_position } & \text { res } & \text { structure } & \text { EDistance } & \text { TDistance } & \text { HDistance } \\ \text { NP_000303.1 } & 1 & \text { M } & \text { other } & 4.05 & 0.01 & 7.35 \\ \text { NP_000303.1 } & 2 & \text { W } & \text { other } & 2.91 & 0.33 & 8.46 \\ \text { NP_000303.1 } & 3 & \text { Q } & \text { other } & 2.92 & 1.09 & 5.14 \\ \text { NP_000303.1 } & 4 & \text { L } & \text { other } & 4.04 & 1.36 & 2.99 \\ \text { NP_000303.1 } & 5 & \text { T } & \text { helix } & 4.47 & 1.90 & 1.04 \\ \text { NP_000303.1 } & 6 & \text { S } & \text { helix } & 3.94 & 3.91 & 0.26 \\ \text { NP_000303.1 } & 7 & \text { L } & \text { helix } & 3.95 & 5.59 & 0.15 \\ \text { NP_000303.1 } & 8 & \text { L } & \text { helix } & 3.92 & 5.62 & 0.15 \\ \text { NP_000303.1 } & 9 & \text { L } & \text { helix } & 3.95 & 5.57 & 0.03 \\ \text { NP_000303.1 } & 10 & \text { F } & \text { helix } & 4.08 & 5.78 & 0.04 \\ \text { NP_000303.1 } & 11 & \text { V helix } & 3.99 & 5.51 & 0.07 \\ \text { NP_000303.1 } & 12 & \text { A helix } & 3.71 & 5.20 & 0.10 \\ \text { NP_000303.1 } & 13 & \text { T } & \text { helix } & 4.12 & 5.16 & 0.32 \\ \text { NP_000303.1 } & 14 & \text { W } & \text { helix } & 4.04 & 4.55 & 0.49 \\ \text { NP_000303.1 } & 15 & G & \text { helix } & 3.82 & 3.46 & 0.89 \\ \text { NP_000303.1 } & 16 & \text { I } & \text { helix } & 3.70 & 2.13 & 1.48\end{array}$




\subsubsection{Patterns of sequence conservation}

Position specific iterative BLAST (PSI-BLAST) is a tool to produce a positionspecific scoring matrix (PSSM). During a PSI-BLAST search, a query sequence is given to BLAST to perform an initial search against the SWISS-PROT sequence database, resulting in multiple sequence alignments of the highest scoring hits based on Blosum62 substitution matrix (Bhagwat and Aravind, 2007). The SWISS-PROT sequence database is used after removal of all low complexity sequences resulting in 208,958 sequences. The Blosum62 matrix is a suitable substitution matrix for detecting weak protein similarities (Henikoff and Henikoff, 1992), and is the default choice in PSI-BLAST. The PSSM is created by calculating position-specific scores for each position in the multiple sequence alignment. This PSSM is then used to perform a second BLAST search, and the search results can be used to further refine the PSSM. The scores in PSSM are based on the frequencies of each amino acid in each position of protein sequence. The PSSM gives the log-odds score for finding a particular matching amino acid in a target sequence. Highly conserved positions have high scores, and weakly conserved positions have low scores.

We utilized PSI-BLAST to capture the conservation pattern information and this information was stored into the local database. For each sequence, each amino acid is represented by 20 numbers. These 20 numbers are the substitution scores of similarity between the observed amino acid at this position and all 20 possible amino acids. Figure 6 shows a portion of the Table that contains the PSSM information in the local database. The more positive the score, the more highly conserved the pattern; the more negative 
Figure 6. A portion of the table that contains the PSSM information for each residue of each protein sequence. Each residue is represented by 20 numbers. The 20 numbers are the substitution scores of similarity between this residue and all 20 amino acids. The more positive score, the more highly conserved; the more negative score, the more weakly conserved. 


$\begin{array}{clllllllllllll}\text { ref_num } & \text { res_position } & \text { res } & \text { A } & \text { C } & \text { D } & \text { E } & \text { F } & \text { G } & \text { H } & \text { I } & \text { K } & \text { L } & \text { M } \\ \text { NP_001521.1 } & 1 & \text { M } & -169 & -221 & -395 & -282 & -68 & -354 & -235 & 50 & -214 & 142 & 815 \\ \text { NP_001521.1 } & 2 & \text { E } & -32 & -252 & 229 & 274 & -366 & -162 & -158 & -361 & -83 & -366 & -270 \\ \text { NP_001521.1 } & 3 & \text { G } & -56 & -303 & -206 & -253 & -400 & 570 & -280 & -423 & -216 & -427 & -330 \\ \text { NP_001521.1 } & 4 & \text { A } & 303 & -212 & -343 & -266 & -189 & -46 & -295 & -83 & -233 & 190 & 528 \\ \text { NP_001521.1 } & 5 & \text { G } & -86 & -297 & 197 & -185 & -378 & 449 & -281 & -271 & -215 & -348 & -285 \\ \text { NP_001521.1 } & 6 & \text { G } & 19 & -268 & -163 & -24 & 101 & 194 & 228 & -201 & -142 & -219 & 113 \\ \text { NP_001521.1 } & 7 & \text { A } & -57 & -322 & 198 & 292 & -353 & -280 & 136 & -118 & 3 & -164 & -229 \\ \text { NP_001521.1 } & 8 & \text { N } & 84 & -315 & 52 & 64 & -398 & -236 & -224 & -334 & 190 & -201 & -262 \\ \text { NP_001521.1 } & 9 & \text { D } & -238 & -456 & 572 & 259 & -470 & -272 & -204 & -439 & 231 & -450 & -361 \\ \text { NP_001521.1 } & 10 & \text { K } & -224 & -439 & 155 & 28 & -431 & -314 & -229 & -376 & 512 & -158 & -268 \\ \text { NP_001521.1 } & 11 & \text { K } & 143 & -369 & 6 & 105 & -430 & -77 & -244 & -375 & 450 & -233 & -278 \\ \text { NP_001521.1 } & 12 & \text { K } & -149 & -454 & -271 & -35 & -270 & -356 & -162 & -417 & 259 & -371 & -300 \\ \text { NP_001521.1 } & 13 & \text { I } & -45 & -310 & -353 & -290 & 273 & -138 & -300 & 179 & -40 & 133 & -18 \\ \text { NP_001521.1 } & 14 & \text { S } & 270 & -308 & 43 & 69 & -433 & -238 & -289 & -336 & -203 & -371 & -312 \\ \text { NP_001521.1 } & 15 & \text { S } & 200 & -248 & 95 & -152 & -372 & -181 & -235 & -345 & -157 & -354 & -281 \\ \text { NP_001521.1 } & 16 & \text { E } & -238 & -455 & 17 & 555 & -422 & -327 & 56 & -427 & 78 & -391 & -309 \\ \text { NP_001521.1 } & 17 & \text { R } & -238 & -404 & -1 & 23 & -62 & 99 & 301 & -372 & 172 & -185 & -265 \\ \text { NP_001521.1 } & 18 & \text { R } & -128 & -401 & -291 & -163 & -356 & -341 & -201 & 7 & 241 & -140 & -223 \\ \text { NP_001521.1 } & 19 & \text { K } & -219 & -442 & -191 & 13 & -468 & -297 & -199 & -448 & 538 & -420 & -306 \\ \text { NP 001521.1 } & 20 & \text { E } & -57 & -404 & -89 & 501 & -422 & -177 & -195 & -404 & 227 & -269 & -300\end{array}$


score, the more weakly conserved. For example, at position 1, the amino acid ' $M$ ' has score of 815 , which means ' $M$ ' is highly conserved at this position 1; ' $L$ ' has score of 142 , which means ' $\mathrm{L}$ ' is also highly conserved at this position, but not as high as ' $\mathrm{M}$ '. ' $\mathrm{A}$ ' has score of -169 , which means ' $A$ ' is weakly conserved at position 1; ' $C$ ' has score of -221 , which means ' $C$ ' is more weakly conserved than ' $A$ ' at this position. When training the SVM, all 20 values are supplied as input feature data for each of the 15 amino acids within the local sequence window.

\subsection{Pattern classification}

\subsubsection{Local sequence window}

A local sequence window is a subsequence of the protein with the positive/negative site as the central amino acid.

The attributes of one residue are closely related to its neighbouring amino acids. When we analyze the properties of an amino acid that may be modified via PTM, we need to also consider the effect of its surrounding residues. Therefore, to classify patterns between positive and negative sites, we analyzed not only the potentially modified residue, but also the local sequence window surrounding the putative PTM site. For example, for each positive/negative site, we took into account the surface accessibility, secondary structure, and PSSM information of its local sequence window, not just the positive/negative site itself. 
The sequence window cannot be too large or too small. A sequence window that is too large will increase the dimensionality of the SVM input feature data tremendously, leading to unnecessary complexity of the SVM and to reduced classification accuracy. A sequence window that is too small may result in the loss of important information. A previous study (Mount, 2004) has recommended a sequence window of 13-17 amino acids to perform pattern searches. Considering that we hypothesize that secondary structure may be an important input feature when trying to recognize hydroxylation sites, it is also important to consider the average length of these elements. Because this window size is within the range of lengths found in $\alpha$ helices ( 5 to 40 residues) and $\beta$ strands ( 5 to 10 residues), then it is expected that the identity of all the amino acids within the window should determine the complete secondary structure found to surround the central amino acid (Mount, 2004).

In the present study, we used a sequence window length of 15 residues with the positive/negative site amino acid at the center. This results in 15 numeric inputs for surface accessibility, 45 numeric inputs for secondary structure $(\mathrm{H}, \mathrm{E}$, and $\mathrm{T}$ for each of 15 amino acids) and 300 numeric inputs for PSSM (20 inputs per amino acid times 15 amino acids); this results in 360 numeric inputs in total for each positive/negative site.

\subsubsection{Removing identical data points}

We have 40 protein hydroxylation targets with 61 positive data points and 1,980 negative data points, or 2,041 data points in total. An analysis of these 2,041 putative sites revealed 228 pairs of identical points, with 222 pairs in the negative set and 6 pairs 
in positive set. The identical points have the same values for all 360 inputs. After removing the identical points, we had 1,813 points in total, with 1,758 negative points and 55 positive points. This 1,813 non-identical data set was used as training data set for support vector machine.

To understand why we removed the identical data points, we should know the concepts of apparent error rate and true error rate. Apparent error rate is the error rate measured when a trained classifier is applied to the same data that was used to train it. The true error rate is the error rate observed when a trained classifier is applied to new data, different from that used to train it. If identical data points exist in the training and testing data sets, then we are reporting the apparent error rate instead of true error rate. Considering that the apparent error rate is known to be optimistically biased (Weiss and Kulikowski, 1991), therefore, it would be erroneous to claim that the trained classifier will continue to perform at this accuracy over new data (Duda et al., 2001).

\subsubsection{Support vector machines}

A support vector machine (SVM) is a method of pattern classification. SVMs are extremely powerful tools for recognizing patterns in high dimensional feature space (consider that 360 inputs used here), thus are widely used in bioinformatics (Boser et al., 1992; Scholkopf and Smola, 2002; Vapnik, 1999). SVMs are characterized by two abilities: 1) for the case where data from the 2 classes are linearly separable, SVMs will find the decision boundary between the 2 classes which maximizes the margin between the decision boundary and the data from each class; and 2) for the case where data from 
the 2 classes are not linearly separable, nonlinear kernels are applied to the input feature data to map the data into another space such that the data becomes linearly separable (Duda et al., 2001).

Consider a binary classification problem in the first case (i.e. linearly separable) as illustrated in Figure 7. Here, an SVM can simply separate the data from the 2 classes with a straight line as shown in Figure 7. The separating line defines a decision boundary such that all points above belong to one class, and all points below belong to another class. This concept can be extended to higher dimensional input feature spaces. For data with $\mathrm{n}$ features, an SVM will construct a hyperplane in $\mathrm{n}$-dimensional space to separate the input data into two classes. The optimal hyperplane has maximum distance from the nearest training data points of two categories, therefore this separating hyperplane maximizes the margin between the two data sets. In order to calculate the margin, two parallel hyperplanes, one on each side of the separating hyperplane are constructed. They are pushed up against the two data sets. When the largest distance to the neighbouring data points of both categories is found, the hyperplane gives good separation (Figure 7). The support vectors are those training data points that define the optimal separating hyperplane. In other words, the support vectors are the most informative points for the classification and also the most difficult points to be classified (Duda et al., 2001).

Now consider the second case where the data from each class are not linearly separable. Here, kernel methods provide sufficient computational power to handle nonlinear classifiers. As shown in Figure 8 (Hill and Lewicki, 2007), the original data are non-linearly separable. The kernel function can transform and rearrange the data points such that they become linearly separable (Hill and Lewicki, 2007). Therefore, the 
Figure 7. A classic example of a support vector machine that solves a binary classification (reproduced from Wikipedia Commons, 2009). The separating hyperplane defines a boundary such that all points above belong to one class, and all points below belong to another class. The support vectors are the training data points that define the optimal separating hyperplane, and are shown on the margin. 


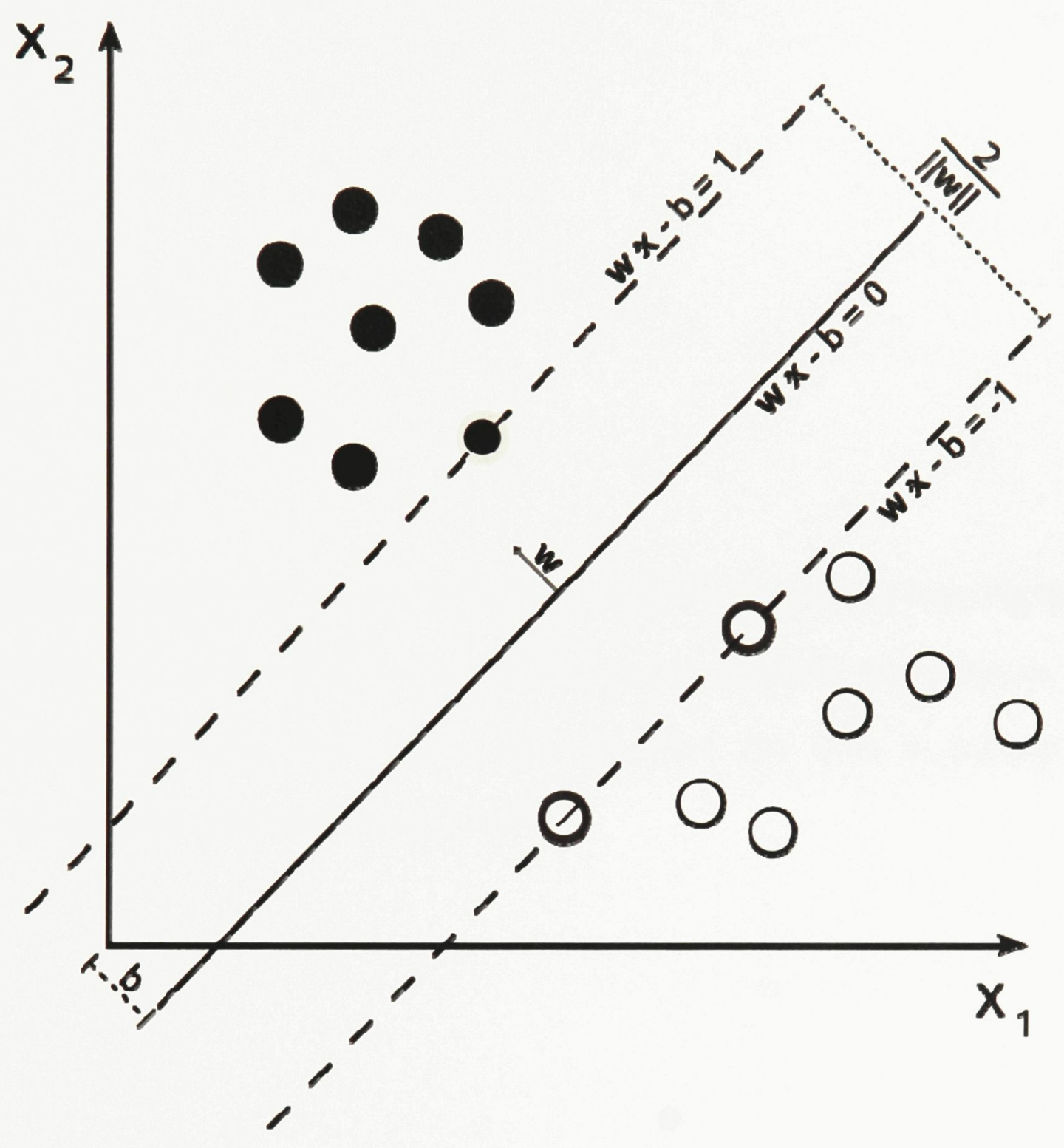


Figure 8. The input data are non-linearly separated (reproduced from Hill and Lewicki, 2007). The kernel function can transform and rearrange the data points to make them linearly separable. Thus kernel functions allow the SVM to perform non-linear classifications (Hill and Lewicki, 2007). 


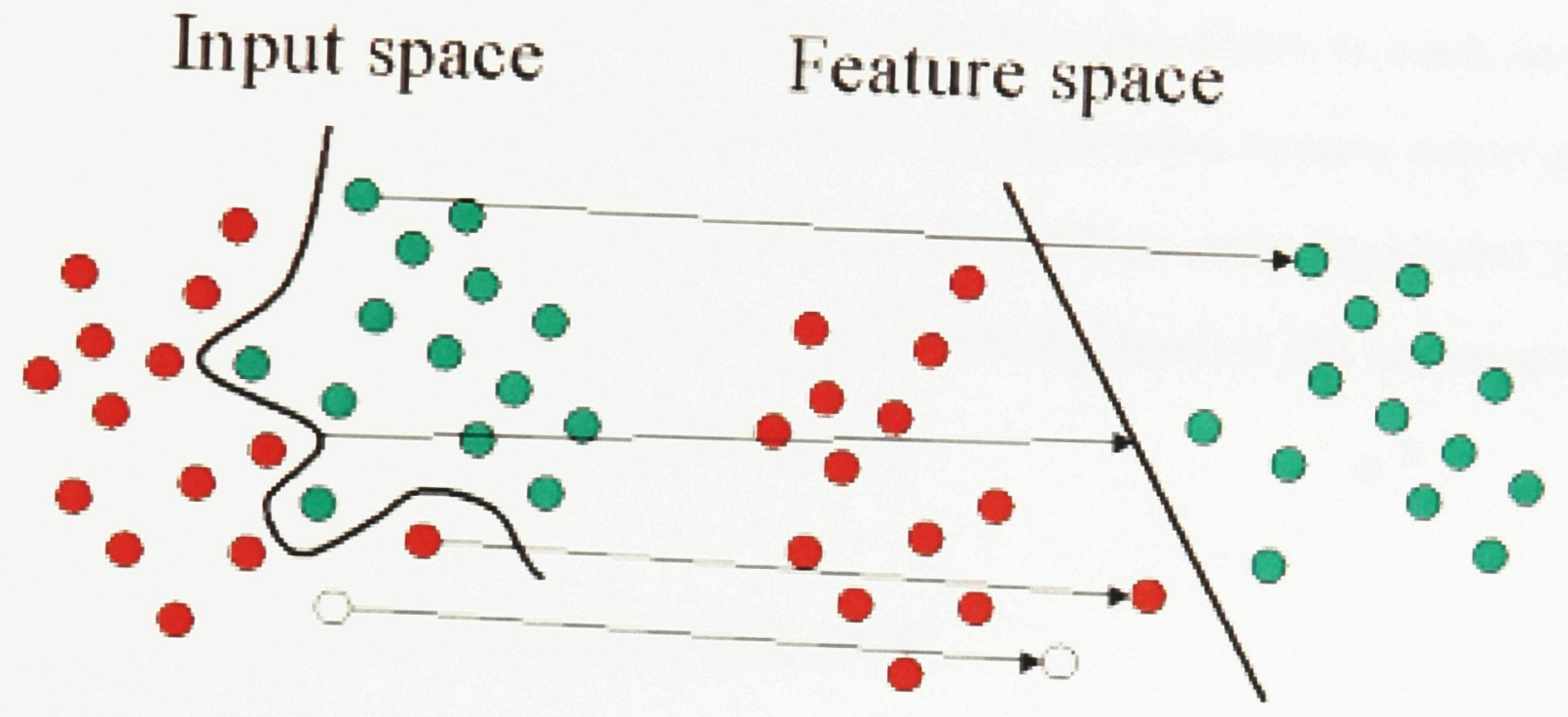


kernel functions allow the SVM to perform non-linear classifications. There are a number of kernels that can be used in SVMs: linear, polynomial, radial basis function (RBF), and sigmoid. RBF is recommended as a reasonable first choice (Hsu et al., 2008) and performed well in the current study. Other kernel functions were only briefly explored and showed no advantage.

LIBSVM is a software library for support vector machines which provides support for several different kernel functions. LIBSVM makes SVMs, as a tool, easy to use (Chang and Lin, 2001). LIBSVM provides a simple interface to users, and let users link it with different programs. The codes for MATLAB are easily downloaded from LIBSVM at http://www.csie.ntu.edu.tw/ cjlin/libsvm/, and installed into any computer. MATLAB was used to train the optimal classifier.

\subsubsection{Analysis of different aspects of the SVM}

A number of aspects of the SVM were explored in order to arrive at the optimal classifier, such as the effect of class imbalance, feature selection, and the creation of subproblems based on the presence of certain protein domains.

Class imbalance means that the number of points in each class is dramatically different. Since we had 1,758 negative points and only 55 positive non-identical points available for training the SVM prediction system, there is a danger that the dominant negative points may overwhelm the information provided by the positive points. One way to address class imbalance is through random undersampling where only a subset of the dominant class is used for training rather than all available data (Sales et al., 2008). We 
explore the impact of class imbalance on SVM prediction accuracy and also explore suitability of random undersampling to deal with class imbalance. As discussed in chapter 8, the use of differentially penalizing the SVM for making errors on the positive vs. negative training examples is ultimately selected as the preferred means to deal with class imbalance.

We have three different features: surface accessibility, secondary structure, and PSSM. We need to find out what the best feature combination is. Therefore, several classifiers will be trained and evaluated using different subsets of the available feature inputs. The inputs of each feature alone, the different pairs of three features, and all three features will be used to train the classifiers. The comparison of these trained classifiers will reveal the best combination of the features.

Amongst our 40 protein targets, we found that a number of them contain either epidermal growth factor (EGF)-type domain or ankyrin repeat domain. EGF-type domains are characterized by the conserved sequence motif $C \cdot[D N] \cdot\{4\}[F Y] \cdot C \cdot C$, where $\{4\}$ means any four amino acides, [DN] means either D or N, and [FY] means either $\mathrm{F}$ or Y. Some EGF domains are post-translationally hydroxylated at the conserved N/D within the sequence motif (Gronke et al., 1989). The ankyrin repeat domain is often involved in protein-protein binding (Sbodio and Chi, 2002), it is not related to the hydroxylation directly. NCBI provides the detailed domain information for all known proteins. I searched our 40 protein targets in NCBI, and found that 22 of them have EGF domains, and 16 of them have ankyrin repeat domain as shown in Table 3. We need to test if these two domains have effect on the classifier. 
Table 3. Amongst the 40 protein targets, 22 proteins contain EGF domain, 16 proteins contain ankyrin repeat domain, 3 proteins contain both domains, and 5 proteins contain neither domain.

\begin{tabular}{|c|c|c|}
\hline Ref_num & EGF domain & $\begin{array}{c}\text { Ankyrin repeat } \\
\text { domain }\end{array}$ \\
\hline NP_000303.1 & Yes & No \\
\hline NP_001724.3 & Yes & No \\
\hline NP_077719.2 & Yes & Yes \\
\hline NP_000304.1 & Yes & No \\
\hline NP_000205.1 & Yes & No \\
\hline NP_060087.2 & Yes & Yes \\
\hline NP_000124.1 & Yes & No \\
\hline NP_000495.1 & Yes & No \\
\hline NP_003882.1 & Yes & No \\
\hline NP_062562.1 & Yes & No \\
\hline NP_002499.1 & Yes & No \\
\hline NP_000122.1 & Yes & No \\
\hline NP_001008390.1 & Yes & No \\
\hline NP_003352.2 & Yes & No \\
\hline NP_958850.1 & Yes & No \\
\hline NP_000426.1 & Yes & Yes \\
\hline NP_001725.1 & Yes & No \\
\hline NP_000352.1 & Yes & No \\
\hline NP_000618.2 & Yes & No \\
\hline NP_055061.1 & Yes & No \\
\hline NP_001399.1 & Yes & No \\
\hline NP_006601.2 & Yes & No \\
\hline NP_003989.2 & No & Yes \\
\hline NP_065390.1 & No & Yes \\
\hline NP_056137.1 & No & Yes \\
\hline NP_002032.2 & No & Yes \\
\hline NP_005245.2 & No & Yes \\
\hline NP_057738.1 & No & Yes \\
\hline NP_057739.1 & No & Yes \\
\hline NP_852092.1 & No & Yes \\
\hline NP_003738.2 & No & Yes \\
\hline NP_002805.1 & No & Yes \\
\hline NP_002471.1 & No & Yes \\
\hline NP_665807.1 & No & Yes \\
\hline NP_060174.2 & No & Yes \\
\hline NP_001521.1 & No & No \\
\hline NP_001421.2 & No & No \\
\hline
\end{tabular}




\begin{tabular}{|c|c|c|}
\hline Q14766 & No & No \\
\hline NP 001398.2 & No & No \\
\hline P00740 & No & No \\
\hline
\end{tabular}


All the results are given below in section 3.2. Later, the optimal classifier was applied to predict novel hydroxylation sites in new protein sequences.

\subsubsection{Evaluation of classification accuracy}

Since we only have 61 positive samples, which is a small amount, we used a resampling technique called the "leave-one-out" test protocol in order to evaluate the performance of our classifier. Assume we have $\mathrm{n}$ points in the training set, we train an SVM on n-1 of them, and test on the single remaining point. This is repeated such that each point in the training set is used once as the test point. This method is termed "leaveone-out" (LOO) and is recommended for cases with limited training data (Weiss and Kulikowski, 1991). To measure the accuracy of the trained classifier, we will utilize a confusion table, also known as a confusion matrix (Duda et al., 2001) (Figure 9).

Each column of the confusion matrix represents the instances in an actual class, and each row represents the instances in a predicted class. The matrix consists of two rows and two columns that report the number of true negatives (TN), false positives (FP), false negatives (FN), and true positives (TP). The FP is type I error and the FN is type II error. From this confusion table, we can calculate the sensitivity, specificity, positive predictive value and Matthew's correlation coefficient (MCC) as the performance metrics to evaluate the trained classifier.

Sensitivity measures the proportion of actual positives, which are correctly identified. Sensitivity is calculated as:

$$
\text { Sensitivity }=T P /(T P+F N)
$$


Figure 9. Confusion matrix (adapted from Duda et al., 2001). Each row of the confusion matrix represents the instances in a predicted class, and each column represents the instances in an actual class. The matrix consists of two rows and two columns that report the number of true negative (TN), false positives (FP), false negatives (FN), and true positives (TP). 


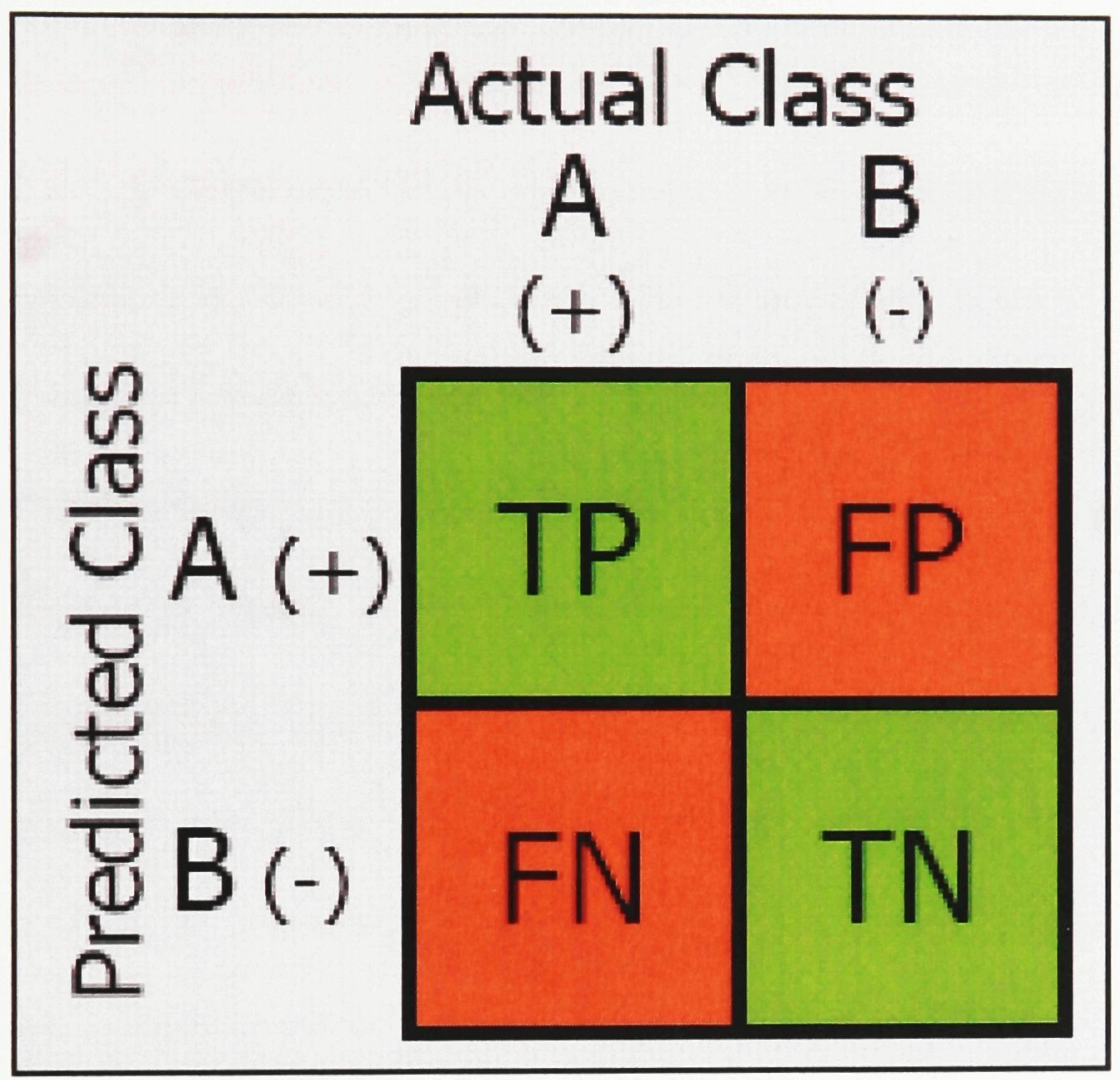


Specificity measures the proportion of negatives, which are correctly identified. Specificity is calculated as:

\section{Specificity $=$ TN $/(T N+F P)$}

Positive predictive value (PPV), also known as precision rate, is the proportion of positives, which are correctly predicted. PPV was calculated as:

$$
P P V=T P /(T P+F P)
$$

MCC combines sensitivity and specificity into a single measure. It returns a value between -1 and +1 . A coefficient of +1 represents a perfect prediction, 0 an average random prediction and -1 an inverse prediction. MCC was calculated as:

$$
M C C=(T P * T N-F P * F N) / s q r t((T P+F P) *(T N+F N) *(T P+F N) *(T N+F P))
$$

Due to gross class imbalance expected in the proteome (eg. most asparagines are not expected to be hydroxylated), it is critical that our specificity be extremely high, even at the expense of sensitivity. The key performance metrics for the final analysis will be positive predictive value and sensitivity. That is, when the prediction tool is run over new data, where the answer is unknown, how certain are we that this is actually a hydroxylation site (actual positive) if the prediction is "yes" (PPV), and if the site is really hydroxylated, how likely is it that our tool will detect it (sensitivity/recall).

\subsection{Developing a web server to predict novel hydroxylation sites}

A web server was developed based on the optimal classifier. It allows users to input their candidate protein sequence and it then returns the results indicating if any N/D within the sequence is likely to be hydroxylated. 


\section{RESULTS}

\subsection{Characterization of known asparagine/aspartate hydroxylation sites}

\subsubsection{Surface accessibility information on the positive sites}

We predicted the surface accessibility of all the 61 positive sites by using RVPNET (Ahmad et al., 2003). As shown in Table 4, with the exception of a single site (residue $\mathrm{N}$ at position 847 of endothelial PSA domain protein 1), all positive sites are exposed. This result is not surprising considering that if the site residue is buried within the folded protein structure, then there is little chance that it would be modified by an external modifying enzyme. However, amino acid residues exposed on the surface of the folded protein have a greater chance of being modified. RVP-NET predicted that close to all potential sites of hydroxylation $(98 \%)$ occurred on the surface of proteins.

\subsubsection{Secondary structure information on the positive sites}

We determined the secondary structure of all the 61 positive sites using PCI-SS. Most positive sites were located in non-regular structure $(52 / 61=85 \%)$, and the remaining sites were located in $\beta$-strand structure $(9 / 61=15 \%)$. None of the site occurred in an $\alpha$-helix, as shown in Table 5. The result showed a trend for potential hydroxylated sites to be located primarily in non-regular structures, and supported the use of secondary structure as input data to the hydroxylation prediction SVM. Figure 10 shows HIF- $\alpha$ 
Table 4. The predicted surface accessibility of 61 positive sites from RVP-NET. With the exception of a single site (residue $\mathrm{N}$ at position 847 of endothelial PSA domain protein 1 shown in bold), almost all positive sites are exposed. E denotes 'exposed', and B represents 'buried'.

\begin{tabular}{|c|c|c|c|c|c|c|c|}
\hline ref_num & $\begin{array}{c}\text { Res } \\
\text { Pos'n }\end{array}$ & Res & E/B & ref_num & $\begin{array}{c}\text { Res } \\
\text { Pos'n }\end{array}$ & res & E/B \\
\hline NP_000303.1 & 113 & $\mathrm{D}$ & E & NP_003989.2 & 679 & $\mathrm{~N}$ & $\mathrm{E}$ \\
\hline NP_001724.3 & 167 & $\mathrm{~N}$ & $\mathrm{E}$ & NP_002805.1 & 100 & $\mathrm{~N}$ & $\mathrm{E}$ \\
\hline NP_077719.2 & 1904 & $\mathrm{~N}$ & $\mathrm{E}$ & NP_077719.2 & 1971 & $\mathrm{~N}$ & $\mathrm{E}$ \\
\hline NP_001521.1 & 803 & $\mathrm{~N}$ & $\mathrm{E}$ & NP_000304.1 & 136 & $\mathrm{D}$ & E \\
\hline NP_000304.1 & 177 & $\mathrm{~N}$ & $\mathrm{E}$ & NP_000304.1 & 219 & $\mathrm{~N}$ & E \\
\hline NP_000304.1 & 258 & $\mathrm{~N}$ & $\mathrm{E}$ & NP_000205.1 & 391 & $\mathrm{D}$ & $\mathrm{E}$ \\
\hline NP_000205.1 & 466 & $\mathrm{D}$ & $\mathrm{E}$ & NP_000205.1 & 504 & $\mathrm{~N}$ & $\mathrm{E}$ \\
\hline NP 000205.1 & 542 & $\mathrm{~N}$ & $E$ & NP 000205.1 & 646 & $\mathrm{D}$ & $\mathrm{E}$ \\
\hline NP 000205.1 & 684 & $\mathrm{D}$ & $\mathrm{E}$ & NP_000205.1 & 799 & $\mathrm{D}$ & $\mathrm{E}$ \\
\hline NP 000205.1 & 837 & $\mathrm{D}$ & $\mathrm{E}$ & NP 002471.1 & 226 & $\mathrm{~N}$ & $\mathrm{E}$ \\
\hline NP_003738.2 & 864 & $\mathrm{~N}$ & $E$ & NP_000352.1 & 342 & $\mathrm{~N}$ & $\mathrm{E}$ \\
\hline NP_060087.2 & 1956 & $\mathrm{~N}$ & $\mathrm{E}$ & NP_060087.2 & 2023 & $\mathrm{~N}$ & E \\
\hline NP_000124.1 & 110 & $\mathrm{D}$ & $\mathrm{E}$ & NP_056137.1 & 526 & $\mathrm{~N}$ & E \\
\hline NP_000495.1 & 103 & $\mathrm{D}$ & $\mathrm{E}$ & NP_057738.1 & 98 & $\mathrm{~N}$ & $\mathrm{E}$ \\
\hline NP_003882.1 & 104 & $\mathrm{D}$ & E & NP_005245.2 & 98 & $\mathrm{~N}$ & E \\
\hline NP_057739.1 & 98 & $\mathrm{~N}$ & $\mathrm{E}$ & NP_002032.2 & 98 & $\mathrm{~N}$ & E \\
\hline NP 852092.1 & 98 & $\mathrm{~N}$ & $\mathrm{E}$ & NP_060174.2 & 168 & $\mathrm{~N}$ & E \\
\hline NP_062562.1 & 101 & $\mathrm{D}$ & $\mathrm{E}$ & NP_002499.1 & 729 & $\mathrm{~N}$ & E \\
\hline NP_002499.1 & 819 & $\mathrm{~N}$ & $\mathrm{E}$ & NP 000122.1 & 123 & $\mathrm{D}$ & $\mathrm{E}$ \\
\hline NP_001008390.1 & 85 & $\mathrm{~N}$ & $\mathrm{E}$ & NP_001008390.1 & 128 & $\mathrm{~N}$ & E \\
\hline NP 003352.2 & 85 & $\mathrm{~N}$ & $\mathrm{E}$ & NP_003352.2 & 128 & $\mathrm{~N}$ & E \\
\hline NP 665807.1 & 62 & $\mathrm{~N}$ & $\mathrm{E}$ & NP 065390.1 & 210 & $\mathrm{~N}$ & E \\
\hline NP_065390.1 & 244 & $\mathrm{~N}$ & E & NP_958850.1 & 149 & $\mathrm{~N}$ & E \\
\hline NP_000426.1 & 1866 & $\mathrm{~N}$ & E & NP_000426.1 & 1933 & $\mathrm{~N}$ & $\mathrm{E}$ \\
\hline NP_001725.1 & 149 & $\mathrm{~N}$ & $\mathrm{E}$ & NP_ 000618.2 & 649 & $\mathrm{~N}$ & E \\
\hline NP_000618.2 & 812 & $\mathrm{~N}$ & $\mathrm{E}$ & NP 055061.1 & 1666 & $\mathrm{~N}$ & E \\
\hline NP_ 055061.1 & 1889 & $\mathrm{D}$ & $\mathrm{E}$ & NP 001399.1 & 1591 & $\mathrm{~N}$ & E \\
\hline NP_001399.1 & 1810 & $\mathrm{~N}$ & $\mathrm{E}$ & NP_001421.2 & 847 & $\mathbf{N}$ & B \\
\hline Q14766 & 848 & $\mathrm{~N}$ & E & Q14766 & 1011 & $\mathrm{~N}$ & $E$ \\
\hline NP_006601.2 & 158 & $\mathrm{~N}$ & $E$ & NP_001398.2 & 1963 & $\mathrm{D}$ & $\mathrm{E}$ \\
\hline P00740 & 110 & $\mathrm{D}$ & $\mathrm{E}$ & & & & \\
\hline
\end{tabular}


Table 5. The predicted secondary structure of the 61 positive sites from PCI-SS. The structure includes $\alpha$-helix, $\beta$-strand and non-regular secondary structure. "Other" denotes non-regular structure. Most positive sites were located in non-regular structures $(52 / 61=85 \%)$, and the remaining sites were located in $\beta$-strand structures $(9 / 61=15 \%)$.

None of the sites occurred in $\alpha$-helix structures.

\begin{tabular}{|c|c|c|c|c|c|c|c|}
\hline ref num & $\begin{array}{c}\text { Res } \\
\text { Pos'n }\end{array}$ & res & SS & ref num & $\begin{array}{c}\text { Res } \\
\text { Pos'n }\end{array}$ & res & SS \\
\hline NP 000303.1 & 113 & $\mathrm{D}$ & other & NP 003989.2 & 679 & $\mathrm{~N}$ & other \\
\hline NP 001724.3 & 167 & $\mathrm{~N}$ & other & NP 002805.1 & 100 & $\mathrm{~N}$ & other \\
\hline NP_077719.2 & 1904 & $\mathrm{~N}$ & other & NP 077719.2 & 1971 & $\mathrm{~N}$ & other \\
\hline NP_001521.1 & 803 & $\mathrm{~N}$ & other & NP_ 000304.1 & 136 & $\mathrm{D}$ & other \\
\hline NP_000304.1 & 177 & $\mathrm{~N}$ & other & NP_000304.1 & 219 & $\mathrm{~N}$ & other \\
\hline NP_000304.1 & 258 & $\mathrm{~N}$ & other & NP_000205.1 & 391 & $\mathrm{D}$ & other \\
\hline NP_ 000205.1 & 466 & $\mathrm{D}$ & other & NP_000205.1 & 504 & $\mathrm{~N}$ & other \\
\hline NP_000205.1 & 542 & $\mathrm{~N}$ & strand & NP_000205.1 & 646 & D & other \\
\hline NP 000205.1 & 684 & $\mathrm{D}$ & other & NP 000205.1 & 799 & $\mathrm{D}$ & other \\
\hline NP 000205.1 & 837 & $\mathrm{D}$ & other & NP 002471.1 & 226 & $\mathrm{~N}$ & other \\
\hline NP_003738.2 & 864 & $\mathrm{~N}$ & other & NP_000352.1 & 342 & $\mathrm{~N}$ & strand \\
\hline NP_060087.2 & 1956 & $\mathrm{~N}$ & other & NP_060087.2 & 2023 & $\mathrm{~N}$ & other \\
\hline NP_000124.1 & 110 & $\mathrm{D}$ & other & NP_056137.1 & 526 & $\mathrm{~N}$ & other \\
\hline NP $\quad 000495.1$ & 103 & $\mathrm{D}$ & strand & NP 057738.1 & 98 & $\mathrm{~N}$ & other \\
\hline NP $\quad 003882.1$ & 104 & $\mathrm{D}$ & strand & NP 005245.2 & 98 & $\mathrm{~N}$ & other \\
\hline NP 057739.1 & 98 & $\mathrm{~N}$ & other & NP_002032.2 & 98 & $\mathrm{~N}$ & other \\
\hline NP_852092.1 & 98 & $\mathrm{~N}$ & other & NP_060174.2 & 168 & $\mathrm{~N}$ & other \\
\hline NP_062562.1 & 101 & $\mathrm{D}$ & other & NP_002499.1 & 729 & $\mathrm{~N}$ & other \\
\hline NP_002499.1 & 819 & $\mathrm{~N}$ & strand & NP_000122.1 & 123 & $\mathrm{D}$ & other \\
\hline NP_001008390.1 & 85 & $\mathrm{~N}$ & strand & NP 001008390.1 & 128 & $\mathrm{~N}$ & strand \\
\hline NP_003352.2 & 85 & $\mathrm{~N}$ & strand & NP 003352.2 & 128 & $\mathrm{~N}$ & strand \\
\hline NP_665807.1 & 62 & $\mathrm{~N}$ & other & NP 065390.1 & 210 & $\mathrm{~N}$ & other \\
\hline NP_065390.1 & 244 & $\mathrm{~N}$ & other & NP_958850.1 & 149 & $\mathrm{~N}$ & other \\
\hline NP_000426.1 & 1866 & $\mathrm{~N}$ & other & NP_000426.1 & 1933 & $\mathrm{~N}$ & other \\
\hline NP_001725.1 & 149 & $\mathrm{~N}$ & other & NP_000618.2 & 649 & $\mathrm{~N}$ & other \\
\hline NP_000618.2 & 812 & $\mathrm{~N}$ & other & NP_055061.1 & 1666 & $\mathrm{~N}$ & other \\
\hline NP_055061.1 & 1889 & $\mathrm{D}$ & other & NP_001399.1 & 1591 & $\mathrm{~N}$ & other \\
\hline NP 001399.1 & 1810 & $\mathrm{~N}$ & other & NP_001421.2 & 847 & $\mathrm{~N}$ & other \\
\hline Q14766 & 848 & $\mathrm{~N}$ & other & Q14766 & 1011 & $\mathrm{~N}$ & other \\
\hline NP_006601.2 & 158 & $\mathrm{~N}$ & other & NP_001398.2 & 1963 & $\mathrm{D}$ & other \\
\hline P00740 & 110 & $\mathrm{D}$ & other & & & & \\
\hline
\end{tabular}


Figure 10. PDB (1H2M) structure of HIF- $\alpha$ (red) being hydroxylated by the Factor Inhibiting HIF asparagine hydroxylase (green) on the N-803 residue of HIF- $\alpha$ (shown in red stick format). 2-Oxoglutarate and iron are shown in pink and yellow respectively. The modification site is found in a region of non-regular secondary structure.

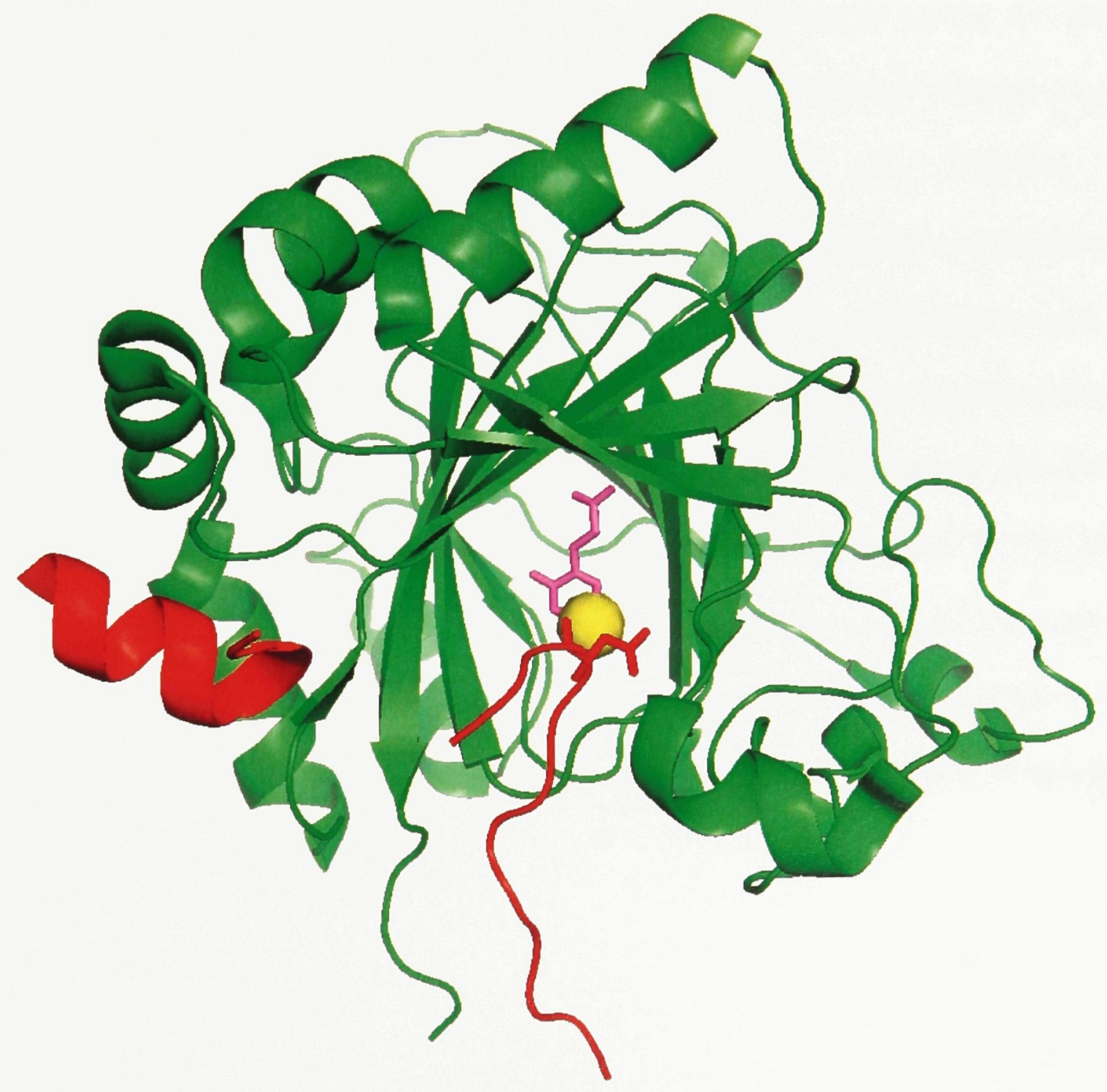


(shown in red) bound to the hydroxylase (shown in green). As can be seen in the figure, the modified amino acid (N-803, shown in red stick format) is located in a non-regular secondary structure state.

\subsubsection{Sequence conservation information on the positive sites}

Figure 11 shows the pattern of the sequence conservation among the local sequence windows of the 61 positive sites illustrated using WebLogo (Crooks et al., 2004). The logo was computed using the online tool available at http://weblogo.berkeley.edu/logo.cgi. It shows some pattern of sequence conservation, such as the prevalence of a) $\mathrm{G}$ being at position 4, b) $\mathrm{C}$ or D being at position 6 , c) $\mathrm{G}, \mathrm{D}$ or $\mathrm{N}$ being at position 11 and d) $\mathrm{C}$ being at position 15 . While sequence logos provide a visual depiction of sequence conservation, they are not suitable for input into the SVM prediction tool since the SVM only can recognize numeric input. Instead, PSI-BLAST with version 2.2.14 is used to compute PSSM data for each sequence in order to capture sequence conservation in numeric form suitable for training the SVM (Altschul et al., 1997). The SWISS-PROT sequence database is used after removal of all low complexity sequences resulting in 208,958 sequences.

\subsection{Pattern classification}

During the process of pattern classification, we used two different datasets for the analysis. The preliminary dataset had 33 protein targets with 51 positive points and 1,290 
Figure 11. The conservation pattern of sequence windows for the 61 positive sites represented as WebLogo (Crooks et al., 2004). The sequence window size is 15 as shown in $\mathrm{X}$-axis. The 61 sequence windows aligned to show the pattern. The $\mathrm{Y}$-axis represents the frequency of the amino acid presented at the window site. The bigger the letter, the more frequent the amino acid presented. The logo shows some conserved patterns, such as the prevalence of a) $\mathrm{C}$ being at position $15, \mathrm{~b}$ ) $\mathrm{C}$ or $\mathrm{D}$ being at position $6, \mathrm{c}$ ) $\mathrm{G}, \mathrm{D}$ or $\mathrm{N}$ being at position 11 and d) $\mathrm{C}$ being at position 15 . 


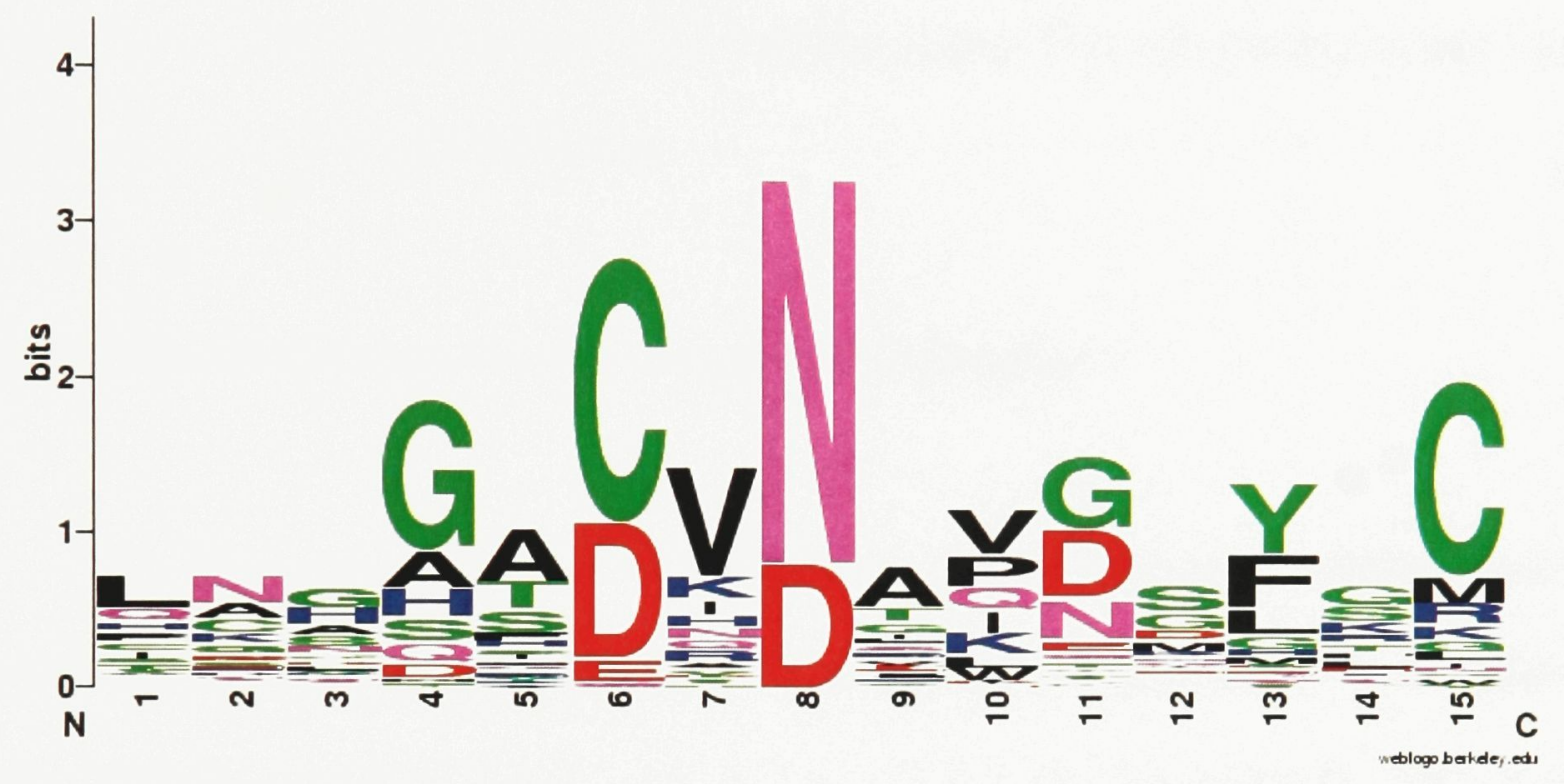


negative points; 1,341 points in total. After removing identical points, the dataset had 1,270 points in total, with 1,223 negative points and 47 positive points. This 1,270 point dataset was used to generate results in section 3.2.1. and 3.2.2 for studying the effect of class imbalance and feature selection.

The final dataset has 40 protein targets with 61 positive points and 1,980 negative points; 2,041 points in total. After removing identical points, the dataset had 1,813 points in total, with 1,758 negative points and 55 positive points. This 1,813 points dataset was used from section 3.2.3 and following.

\subsubsection{The effect of class imbalance on the SVM classifier}

To study the effect of class imbalance, and to determine whether random undersampling is an effective approach, leave-one-out testing was run with different ratios of positive/negative dataset such as $1: 1,1: 2,1: 3$, etc. For example, when of the ratio of the positive/negative points was $1: 1$, the training dataset contained of all 47 positive points and of 47 negative points drawn randomly without replacement from all 1223 negative points. LOO tests for each ratio were repeated 50 times and the average accuracy is reported in order to provide a better estimate of classification accuracy. The classifier was trained on the data of surface accessibility, secondary structure, and PSSM. The result is shown in Figure 12. It shows that sensitivity and PPV decrease with the increase of the positive/negative ratio, revealing a negative effect of class imbalance on the trained SVM classifier. 
Figure 12. The effect of positive/negative ratio to the classifier trained using all 3 features of PSSM, surface accessibility and secondary structure. Leave-one-out was run with different ratios of positive/negative dataset as 1:1, 1:2, 1:3, 1:4, etc. Runs for each ratio were repeated 50 times to reduce the variability of the performance estimate. The error bars indicate the standard deviation. In each run, the negative points are uniquely and randomly chosen from all the negative points. For example, when we run 50 times of LOO for positive/negative dataset as 1:1, the training dataset are 47 positive points and 47 negative points. The 47 negative points are randomly and uniquely chosen from all 1,223 negative dataset. 


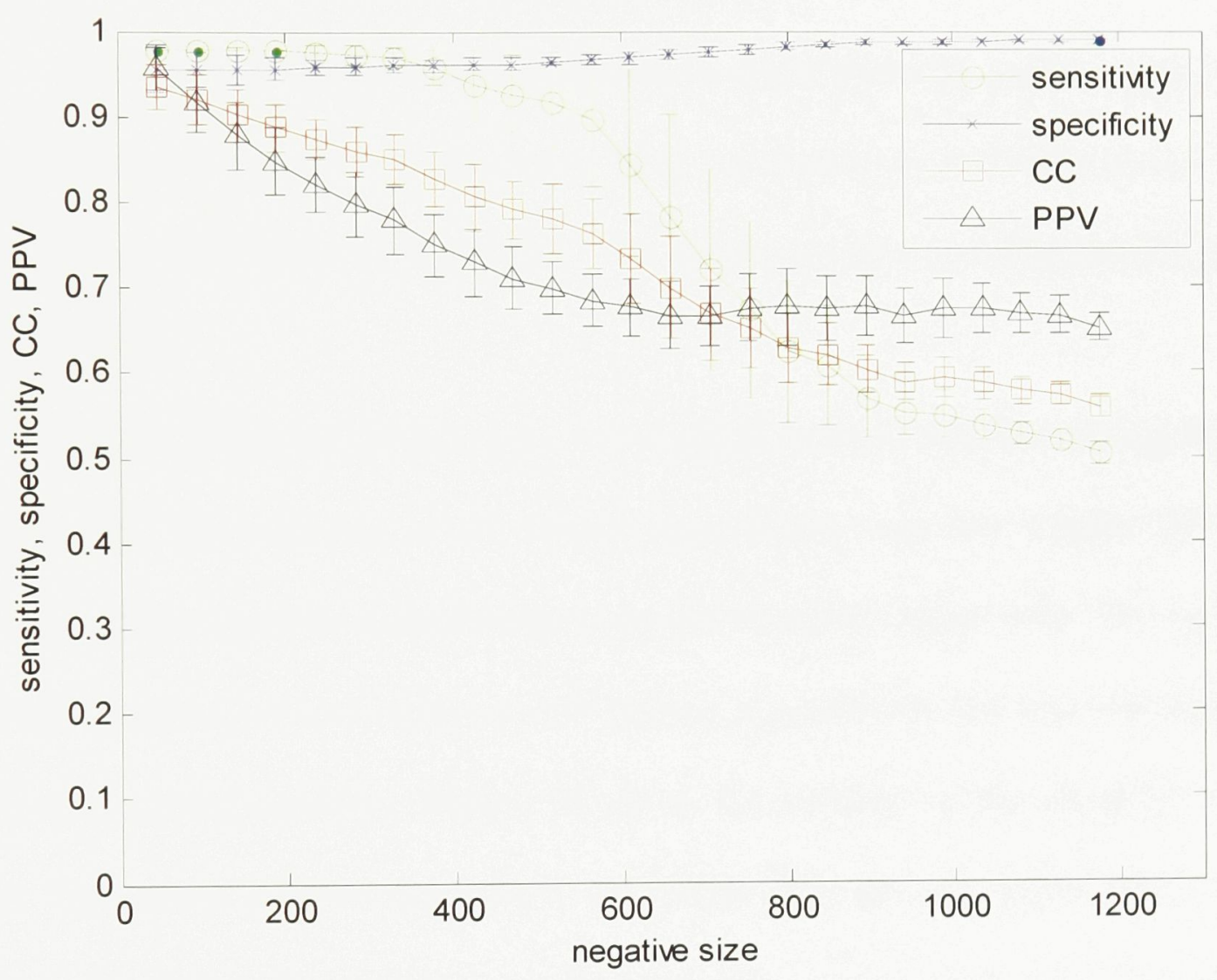




\subsubsection{The effect of feature selection on the SVM classifier}

Another issue with classifier design is feature selection. In the present study we have three different features: surface accessibility, secondary structure, and PSSM. To identify the best feature combination to use as input to the SVM prediction system, I tried different feature inputs in isolation and in combination.

Several experiments were conducted to simultaneously explore class imbalance and feature selection. The experiments were conducted as described above in section 3.2.1., but with different feature inputs. The results are shown in Figures 12, 13, 14, 15, 16, 17 and 18.

Figure 13 shows the accuracy of a classifier trained with the surface accessibility feature only. The accuracy of the surface accessibility was low. Figure $\mathbf{1 4}$ shows the accuracy of the classifier trained with secondary structure feature only. The accuracy was an improvement over that obtained with surface accessibility, but was still insufficient to place it as a good classifier. Figure $\mathbf{1 5}$ shows the accuracy of the classifier with PSSM only. The results showed that PSSM was better than the previously tested classifiers. Figure 16 shows the accuracy of the classifier trained with two features: surface accessibility and secondary structure. The accuracy was low, but better than secondary structure alone. Figure 17 shows the accuracy of the classifier trained with two features: PSSM and surface accessibility. The accuracy was better than the surface accessibility alone, but worse than PSSM alone. Figure 18 shows the accuracy of the classifier trained with two features: PSSM and secondary structure. The accuracy was better than both PSSM alone and secondary structure alone. Figure 12 shows the accuracy of the 
Figure 13. The effect of positive/negative ratio to the classifier trained with surface accessibility feature only. The same protocol was described in Figure 12 was also used here. The PPV and CC points beyond the first point were not available, because PPV and $\mathrm{CC}$ could not be calculated due to a divide-by-zero error. 


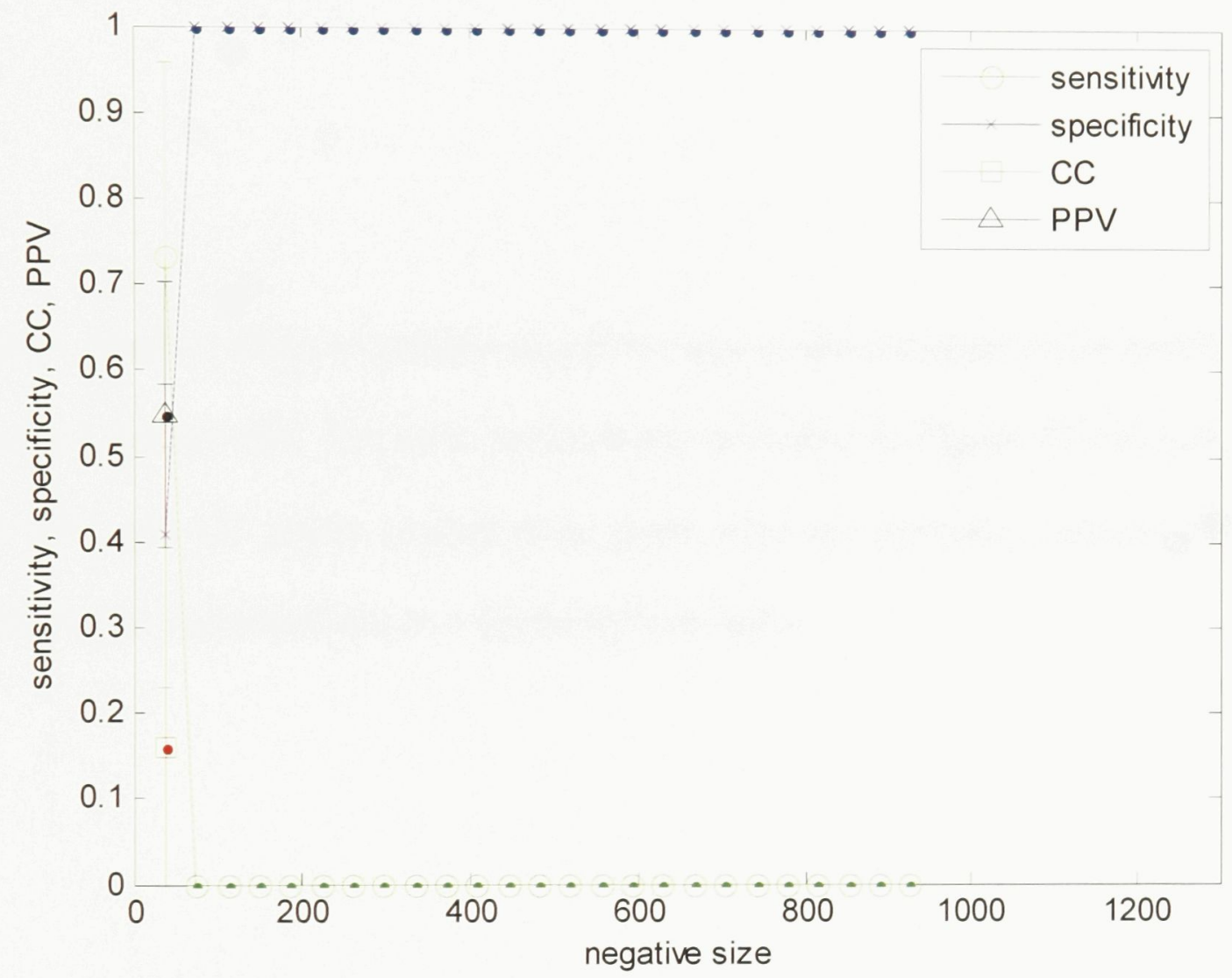


Figure 14. The effect of positive/negative ratio to the classifier trained with secondary structure feature only. The same protocol was described in Figure 12 was also used here. The PPV and CC points beyond some point were not available, because PPV and CC could not be calculated due to a divide-by-zero error. 


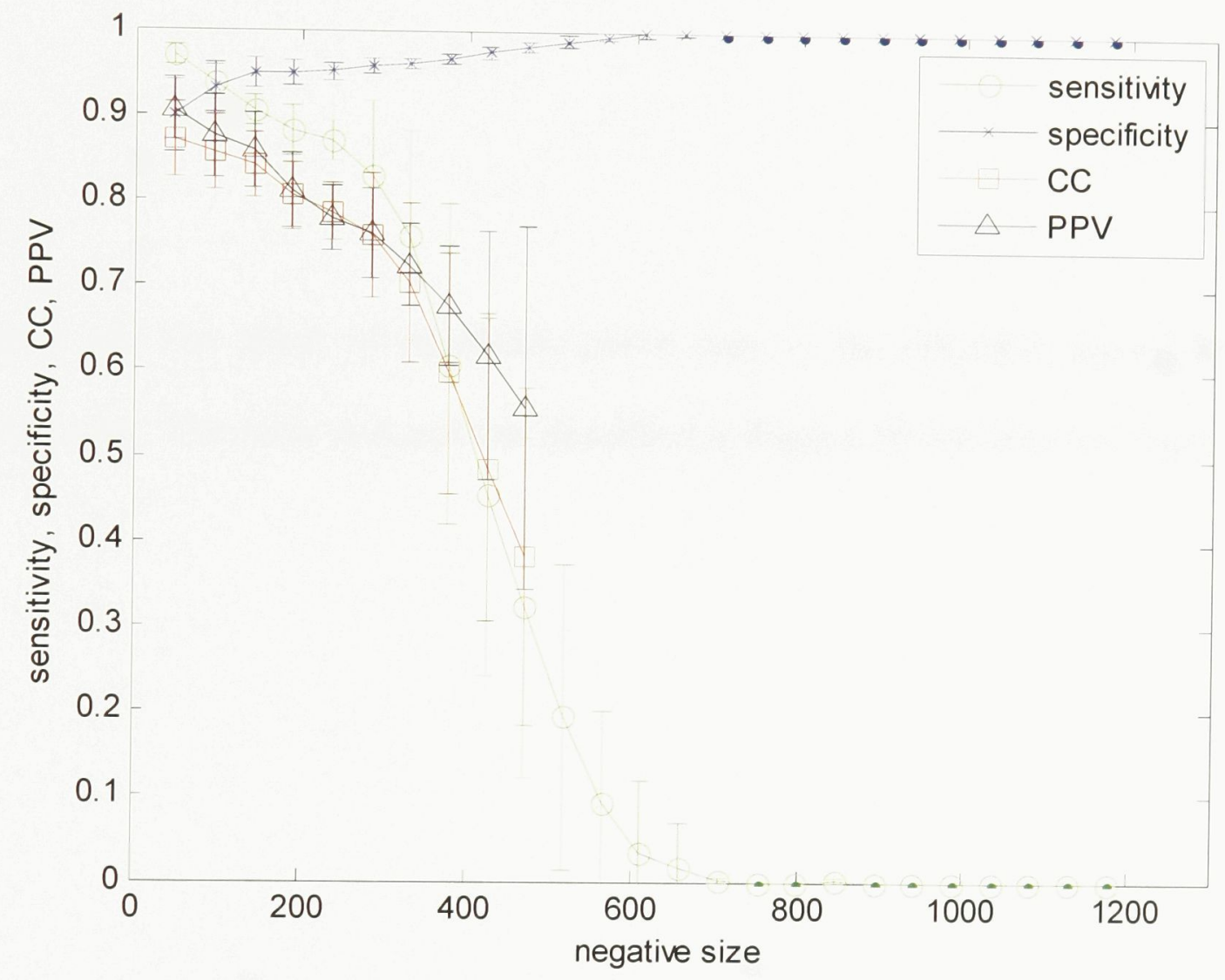


Figure 15. The effect of positive/negative ratio to the classifier trained with PSSM feature only. The same protocol was described in Figure 12 was also used here. 


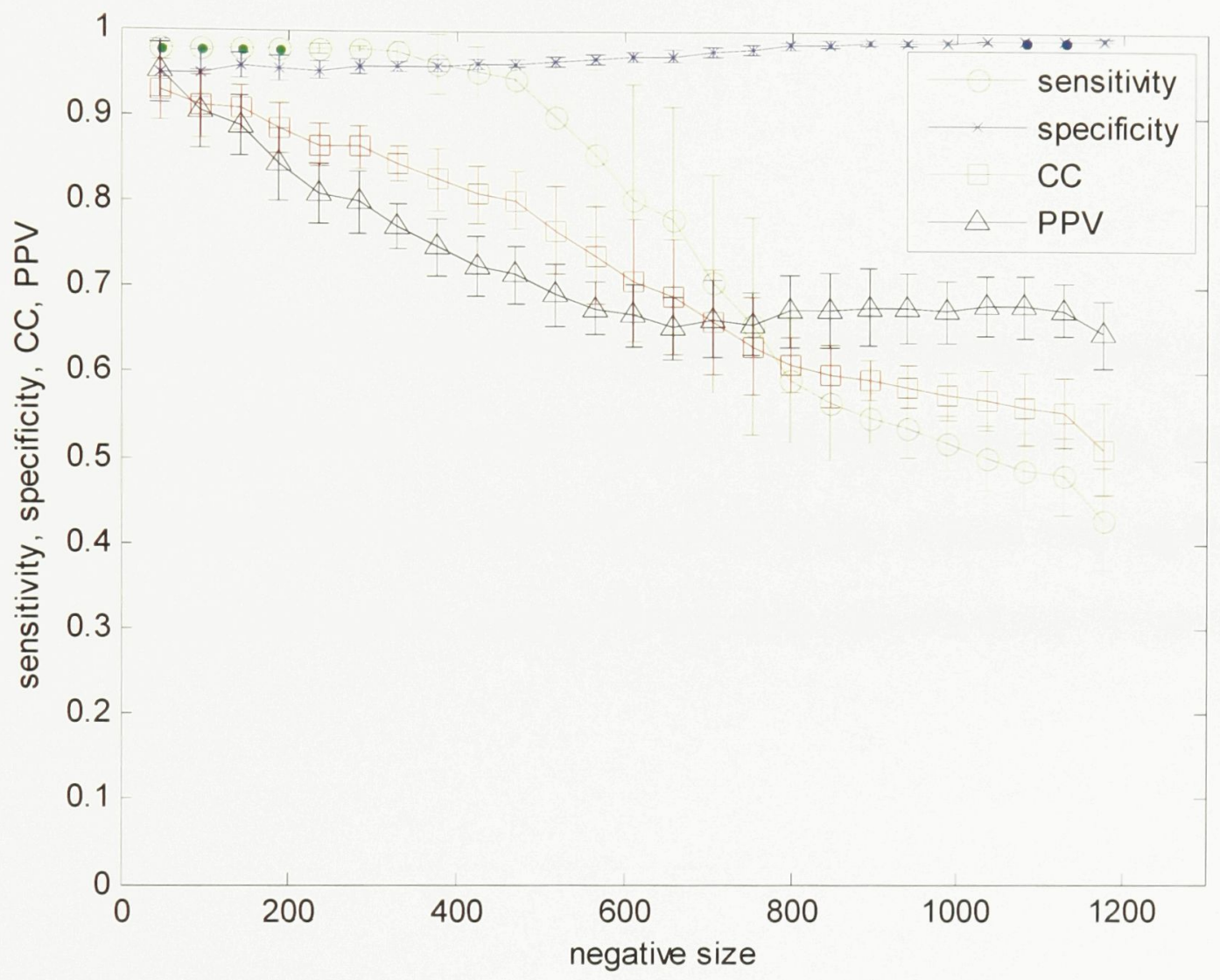


Figure 16. The effect of positive/negative ratio to the classifier trained with surface accessibility and secondary structure features. The same protocol was described in Figure 12 was also used here. The PPV and CC points beyond some point were not available, because PPV and CC could not be calculated due to a divide-by-zero error. 


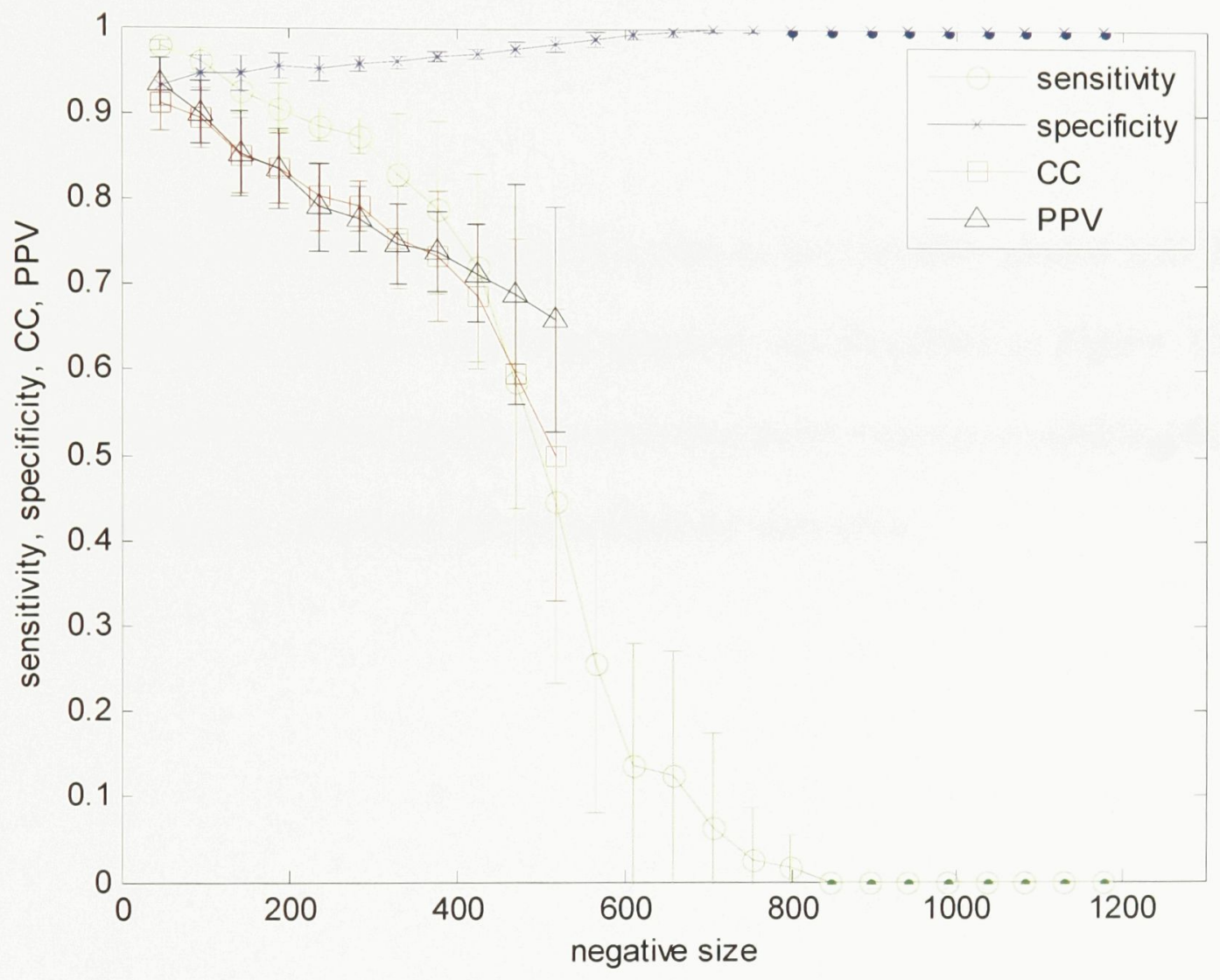


Figure 17. The effect of positive/negative ratio to the classifier trained with PSSM and surface accessibility features. The same protocol was described in Figure $\mathbf{1 2}$ was also used here. The PPV and CC points beyond some point were not available, because PPV and $\mathrm{CC}$ could not be calculated due to a divide-by-zero error. 


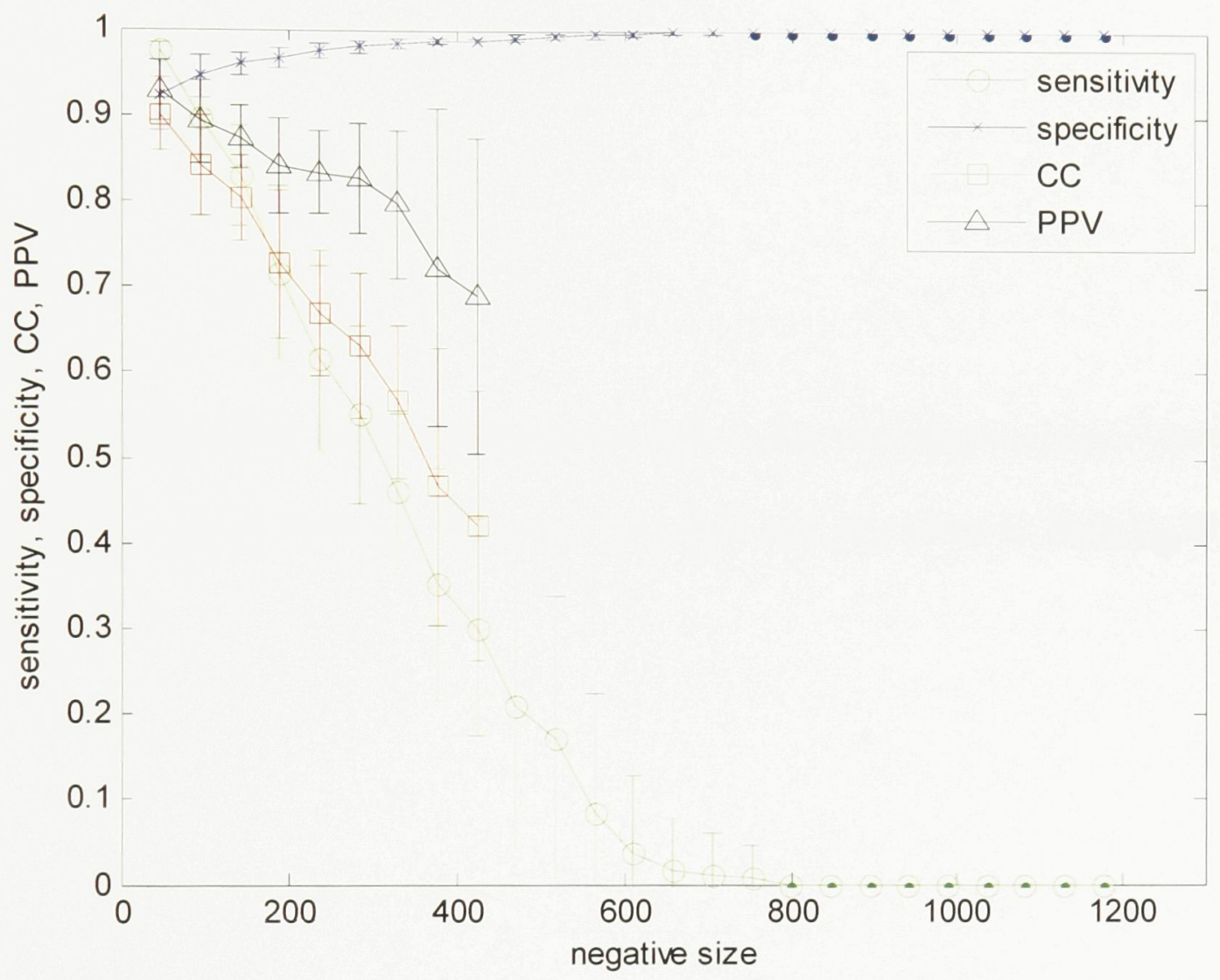


Figure 18. The effect of positive/negative ratio to the classifier trained with PSSM and secondary structure features. The same protocol was described in Figure $\mathbf{1 2}$ was also used here. 


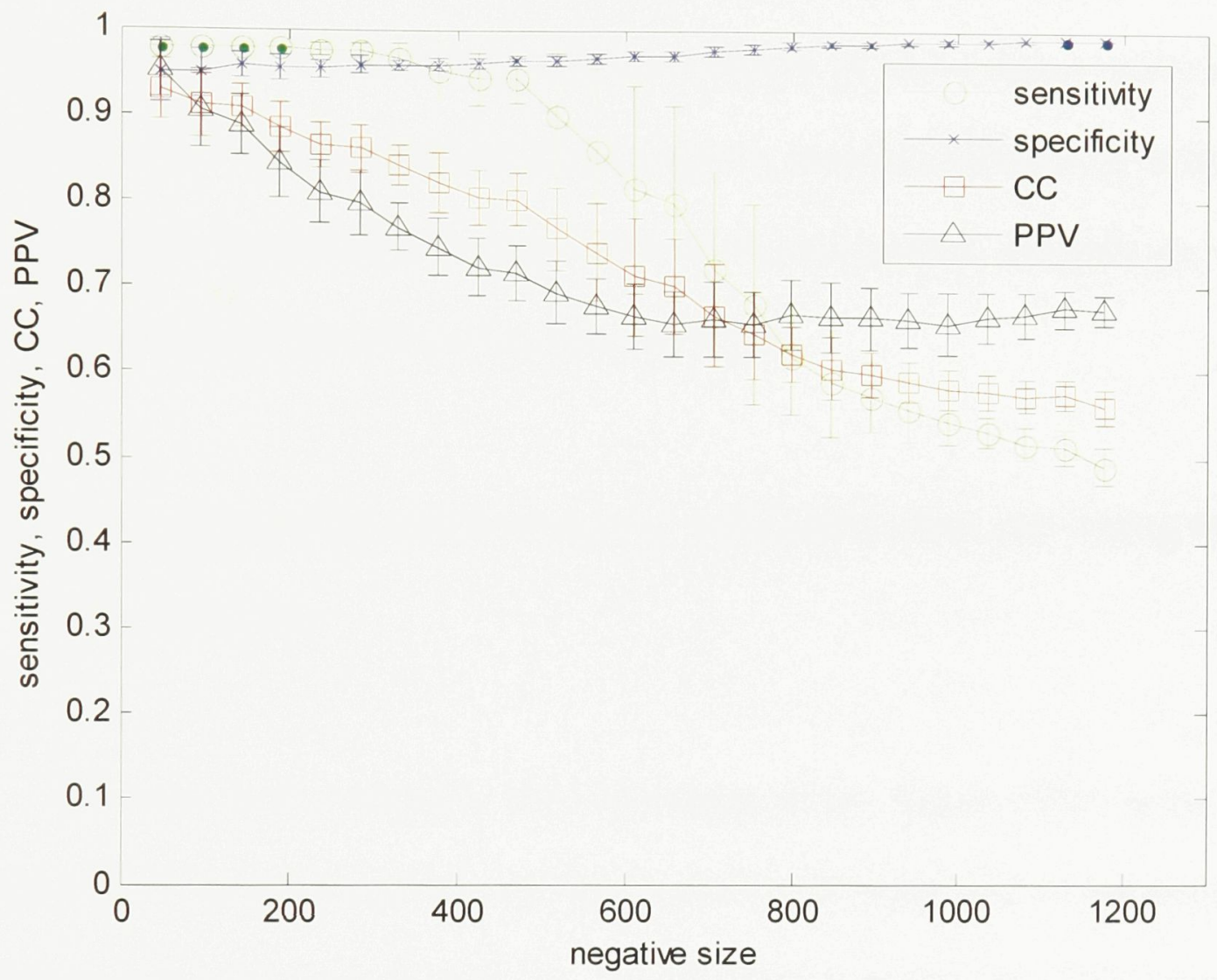


classifier with all three features. It shows that the use of three features provides the most accurate predictions.

The results show that PSSM is the most important feature, followed by secondary structure. Surface accessibility was not very informative, according to tests. When comparing Figure 14 (trained with secondary structure only) to Figure 16 (trained with surface accessibility and secondary structure), Figure $\mathbf{1 6}$ shows better performance than

Figure 14, which indicates that surface accessibility provides information to the classifier as well. The best combination included all three features (surface accessibility, secondary structure and PSSM) and was used to train the SVM.

\subsubsection{Using differential positive/negative misclassification cost ratios to address class imbalance}

Since the positive/negative class imbalance clearly had an effect on the classifier, different SVM positive/negative misclassification cost ratios (one of parameters of the LIBSVM tool), were used to train the classifier on all of the data. This parameter can force the classifier to pay more attention to the smaller class (positive) without having to resort to random undersampling. For example, with ratio of 1:1, the classifier pays equal attention to both positive and negative data; with ratio of 2:1, the classifier pays double attention to errors made on the positive training data compared to the negative data. Thus, we can use all our non-identical 1,813 data set to train the classifier without overwhelming with the negative data. The testing method is still "leave-one-out". Table 6 shows the results. Since sensitivity and PPV are the key performance metrics, the 
Table 6. The leave-one-out test accuracy of the classifiers trained on all of the data of 40 protein targets (1,813 point data set) with different positive/negative SVM misclassification costs, including ratios of $1: 1,2: 1,3: 1,4: 1$. The accuracy is represented by sensitivity (Sn), specificity (Sp), Matthews' correlation coefficient (CC) and positive predictive value (PPV).

\begin{tabular}{|c|c|c|c|c|}
\hline +/- ratio & Sn & Sp & CC & PPV \\
\hline $\mathbf{1 : 1}$ & $49.09 \%$ & $99.26 \%$ & $56.46 \%$ & $67.5 \%$ \\
\hline $\mathbf{2 : 1}$ & $80 \%$ & $98.75 \%$ & $72.11 \%$ & $66.67 \%$ \\
\hline $\mathbf{3 : 1}$ & $92.73 \%$ & $98.18 \%$ & $74.6 \%$ & $61.45 \%$ \\
\hline $\mathbf{4 : 1}$ & $92.73 \%$ & $97.9 \%$ & $72.33 \%$ & $57.95 \%$ \\
\hline
\end{tabular}


positive/negative ratio of $3: 1$ had the best accuracy with a sensitivity of $92.73 \%$ and a PPV of $61.45 \%$. The positive/negative ratio of $3: 1$ was therefore used in all subsequent SVM training. The exclusive testing method was "leave-one-out".

\subsubsection{The effect of domains on the SVM classifier}

Amongst our 40 protein targets, 22 of them have EGF domains containing the motif sequence. Since the EGF domain is a significant characteristic in our protein targets, we want to see if we can improve our classifier based on EGF domain information. That is, if we train separate SVM classifiers for EGF and non-EGF proteins, can better overall classification accuracy be obtained? The 40 protein targets were separated into 2 datasets. One dataset contained the 22 EGF domain-containing proteins. A second dataset contained the 18 proteins without the EGF domain. Using LOO testing, we trained and evaluated separate classifiers with these 2 datasets. The accuracy of the trained classifiers, as well as the overall accuracy, is shown in Table 7. This result will be compared in the following section.

We found the ankyrin repeat domain is another significant characteristic in our protein targets. Of the 40 target proteins, we found that 16 of them have the ankyrin domain. To study the effect of ankyrin domain, I separated the data into 16 ankyrin domain containing proteins, with 633 (19 positive/614 negative) data points and 24 non-ankyrin domain containing proteins, with 637 (28 positive/609 negative) data points. We trained 2 classifiers with these 2 datasets. The accuracy of the trained classifiers, as well as the 
Table 7. The effect of EGF domain on the trained classifier. The 40 protein targets are separated into two groups: one group contained 22 EGF-containing proteins, another group contained 18 proteins without EGF domain. Two classifiers were trained with these two group datasets. The overall accuracy was calculated as well. The accuracy is represented by sensitivity, specificity, CC and PPV.

\begin{tabular}{|l|c|c|c|c|}
\hline & Sn & Sp & CC & PPV \\
\hline EGF containing & $91.67 \%$ & $98.09 \%$ & $71.94 \%$ & $57.89 \%$ \\
\hline Non-EGF containing & $78.95 \%$ & $97.58 \%$ & $62.49 \%$ & $51.72 \%$ \\
\hline Overall & $87.27 \%$ & $97.93 \%$ & $68.72 \%$ & $55.81 \%$ \\
\hline
\end{tabular}


Table 8. The effect of ankyrin repeat domain on the trained classifier. The 40 protein targets are separated into two groups: one group contained 16 ankyrin repeat domaincontaining proteins, another group contained 24 proteins without ankyrin repeat domain. Two classifiers were trained with these two group datasets. The overall accuracy was calculated as well. The accuracy is represented by sensitivity, specificity, CC and PPV.

\begin{tabular}{|l|c|c|c|c|}
\hline & Sn & Sp & CC & PPV \\
\hline Ankyrin containing & $100 \%$ & $97.72 \%$ & $75.01 \%$ & $57.58 \%$ \\
\hline Non-ankyrin containing & $83.78 \%$ & $98.79 \%$ & $73.54 \%$ & $65.96 \%$ \\
\hline Overall & $89.29 \%$ & $98.45 \%$ & $73.86 \%$ & $62.5 \%$ \\
\hline
\end{tabular}


overall accuracy, is shown in Table 8. This result will be compared in the following section.

\subsubsection{The optimal and final classifiers}

A comparison of the classifiers trained on original dataset, EGF domain-based dataset, and ankyrin repeat domain-based dataset is shown in Table 9. There was no significant difference between datasets. We determined that the optimal classifier is the one that is trained on the original dataset, which can recall $92.73 \%$ asparagine/aspartate hydroxylation sites with $61.45 \%$ precision. Considering the relatively small size of our data (i.e. only 61 positive sites), it is important to consider the expected variance on our performance estimates. Computing a 95\% confidence interval on the observed accuracy, results in a sensitivity of $82.4-98.0 \%$ and a precision of $50.1-71.9 \%$ (Duda et al., 2001).

In order to report the accuracy of the classifier, we run different experiments with LOO test method. Since the optimal classifier we got was trained on the original dataset and used positive/negative ratio of 3:1. The final classifier was trained with all the 2,041 points data set with positive/negative ratio of $3: 1$, and was used to predict novel protein sequence. By running all training data back through the final classifier, we can obtain an estimate for the apparent error rate. While this is known to be an optimistically biased measure of performance, it is interesting nonetheless. Of the 61 positive sites, the fully trained final classifier was able to recognize 60 of them, resulting in a sensitivity that was 60/61 (91.2 - 99.9\% confidence interval). 
Table 9. Comparison of different trained classifiers. This table shows the accuracy of the classifier trained on all data (original dataset), and the classifiers trained based on EGF domain and ankyrin repeat domain datasets. The accuracy is measured by sensitivity, specificity, CC and PPV.

\begin{tabular}{|l|c|c|c|c|}
\hline & Sn & Sp & CC & PPV \\
\hline Original dataset & $92.73 \%$ & $98.18 \%$ & $74.6 \%$ & $61.45 \%$ \\
\hline EGF based dataset & $87.27 \%$ & $97.93 \%$ & $68.72 \%$ & $55.81 \%$ \\
\hline Ankyrin based dataset & $89.29 \%$ & $98.45 \%$ & $73.86 \%$ & $62.5 \%$ \\
\hline
\end{tabular}




\subsection{The hydroxylation prediction web server}

After determining our final classifier, we developed a web-based user-friendly interface to make this tool available to the research community at large. The interface allows the user to input any candidate protein sequence and receive the predicted results indicating if each N/D in the candidate protein has the potential to be hydroxylated. Custom software was written in several languages including PHP, PERL, and HTML in order to automate the analysis pipeline and create the web interface. A stand-alone $\mathrm{C}++$ implementation of LIBSVM was used to run the trained SVM on new sequence data input by the user. Figure 19 shows a screen shot of the web page that allows user to input the candidate protein sequence. When a user submits a sequence, the web server will automatically compute the numeric data of surface accessibility, secondary structure, PSSM for this input sequence and generate the test data set. The final SVM classifier is then applied to the computed feature data, and returns the prediction results to the user. Figure 20 shows a screen shot of part of the web page that returns the predicted results.

The tool - HYPT is available at http://bioinf.sce.carleton.ca/HYPT. The web server is running on a dual-Opteron server. It takes approximately one minute to analyze one protein with a length of 800 amino acids, and runtime varies with protein length. The vast majority of runtime is due to PSI-BLAST computing the PSSM data. 
Figure 19. The screen shot of the interface of the prediction web service. It allows user to input their candidate protein sequence. 
Carleton Systems and Computer

Engineering

\title{
HYDROXYLATION
}

\section{Asparagine/aspartate Hydroxylation Site Prediction Server}

\author{
J.R. Green, W.G. Willmore, and Z. Liu
}

$\underline{\text { James Green }}$

William Willmore

Help
Description Hydroxylation is one type of oxygen-dependent post translation modification (PTM). Inhibition of hydroxylation can mimic the hypoxic environment, initiating cellular defense mechanisms for the survival of severe hypoxic conditions. Proteins that can by hydroxylated are therefore potential drug targets for cellular hypoxia survival and the minimization of damage resulting from the hypoxic condition. Experimental approaches are expensive and time-consuming. Therefore computational prediction tool to narrow the protein targets for experimental validation is needed

We developed a prediction tool for asparagine/aspartate hydroxylation. This tool used support vector machine to train on the experimentally verified protein targets. The features we used were position specific scoring matrix (PSSM), secondary structure, and surface accessibility

\section{Sequence}

Name

? Sequence
Submit Clear Form Use Sample Data

References PCI-SS - Green, J.R., Korenberg, M.J., Aboul-Magd, M.O. "PCI-SS: MISO dynamic nonlinear protein secondary structure prediction," BMC Bioinformatics 10 : 222,2009 .

PSIPRED - Jones, D.T. "Protein secondary structure prediction based on positionspecific scoring matrices," J. Mol. Biol. 292: 195-202, 1999

RVP-NET - Ahmad, S., Gromiha, M.M., Sarai, A. "Real Vahe Prediction of Solvent Accessibility From Amino Acid Sequence," Proteins, vol. 50, pp. 629-635, 2003.

$$
2009
$$


Figure 20. The screen shot of part of the web page that returns the predicted results. It shows whether each N/D residue within the input sequence is predicted to be hydroxylated or not. "NO" means this site is non-hydroxylated, "YES" means this site has potential to be hydroxylated. The Confidence tells that how certain we are to predict "YES" or "NO". 


\section{- Carleton Syspartment af and computer Engineering}

\section{HYDROXYLATION}

Asparagine/aspartate Hydroxylation Site Prediction Server

J.R. Green, W.G. Willmore, and Z. Liu

\begin{tabular}{c|ccccc} 
James Green & Results & Position & Residue & $\begin{array}{c}\text { Hydroxylated/Non- } \\
\text { Wydroxylated }\end{array}$ & $\begin{array}{c}\text { Confidence } \\
\text { William Willmore }\end{array}$ \\
& 8 & N & NO & $99.2893 \%$ \\
& 9 & D & NO & $99.4108 \%$ \\
& 24 & D & NO & $99.3707 \%$ \\
& 49 & N & NO & $99.1758 \%$ \\
55 & D & NO & $99.1416 \%$ \\
& 74 & D & NO & $98.8819 \%$ \\
& 77 & D & NO & $99.202 \%$ \\
& 79 & D & NO & $98.5375 \%$ \\
& 82 & D & NO & $99.2646 \%$ \\
& 83 & D & NO & $99.2312 \%$ \\
& 89 & N & NO & $98.8138 \%$
\end{tabular}




\section{DISCUSSION}

\subsection{Summary of contributions}

\subsubsection{Data collection and storage}

dbPTM includes all of the experimentally validated PTM sites from Swiss-Prot, PhosphoELM and O-GLYCBASE. However, it only listed 19 proteins targets for asparagine/aspartate hydroxylation sites. A thorough review of the literature revealed 21 new protein targets that dbPTM had not identified. This successful data collection established the foundation for this research.

A local database was created to store the protein sequence of all human proteins, all the positive and negative site locations on all protein targets for asparagine/aspartate hydroxylation, and the three feature values for all residues of all protein targets. The stored information was queried to produce input feature data for the SVM training. The local database also includes the surface accessibility value of each residue in each sequence for all human proteins, the secondary structure values of each residue in each sequence for all human proteins, and the PSSM values of each residue in each sequence for all human proteins. Therefore, this database is not only useful for this research, but also could be utilized to develop similar programs for further related studies on human protein. 


\subsubsection{Characterizing target proteins}

The protein targets identified during the literature review were characterized by three features: surface accessibility, secondary structure, and pattern of sequence conservation. We computed the surface accessibility with RVP-NET, computed the secondary structure with PCI-SS, and computed PSSM with PSI-BLAST. These feature extraction methods could be useful for future similar studies of other PTMs.

\subsubsection{Pattern classification}

We used an SVM to train an optimal classifier that can recall $92.73 \%$ of all asparagine/aspartate hydroxylation sites with $61.45 \%$ precision, as measured with a leave-one-out test protocol. The accuracy is quite good and is expected to be sufficient for useful application in a biology lab.

Before the development of our prediction tool, the only way to identify possible N/D hydroxylation sites was to find out if a given protein contained the EGF domain. The conserved asparagine/aspartate in the motif would be considered the candidate site to test. Amongst our all 2,041 sites, 62 sites are located in an EGF domain motif, and only 31 of them are experimentally validated as being hydroxylation sites. Using this technique, one would achieve a sensitivity of $31 / 61=50.8 \%$ and a PPV/precision of $31 / 62=50 \%$. Our classifier's sensitivity (92.7\%) and precision (61.5\%) has much higher accuracy. Thus the asparagine/aspartate hydroxylation site prediction tool provides a significant improvement in making accurate predictions. 


\subsubsection{The hydroxylation prediction web server}

We developed a prediction web server for asparagine/aspartate hydroxylation, and made it available to research community. Since there is no existing tool for asparagine/aspartate hydroxylation, our tool would be a milestone for the researchers who are interested in this type of hydroxylation.

\subsection{Potential improvement}

\subsubsection{Training dataset}

The most prominent feature of our classifier is the training dataset. Through dbPTM and in-depth literature review, we found as many as possible of the protein targets with 61 positive points and 1,980 negative points. This had a number of implications to the training as outlined below.

First, the positive dataset utilized came from experimentally verified protein targets from the literature, of which there were few examples. To explore the dependency of SVM accuracy on training set size, "leave-one-out" tests were run with increasing training set size. Balanced training sets were used, starting from 5 positive/5 negative, 10 positive/10 negative, 15 positive/15 negative, etc, and each test was repeated 100 times for each size. Figure 21 clearly shows that the accuracy of the classifier increases with increasing size of the training set with a plateau occurring with a training set size of 30 . 
Figure 21. The effect of different training set size to the classifier trained on surface accessibility, secondary structure and PSSM features. Leave-one-out was run with balanced dataset of increasing size, starting from 5 positive $/ 5$ negative, 10 positive/10 negative, etc. (run 100 times for each size). In each run, the positive/negative training sets were uniquely and randomly drawn from the whole dataset. The accuracy of the classifier increases with increasing size of the training set with a plateau occurring with a training size of 30 . 


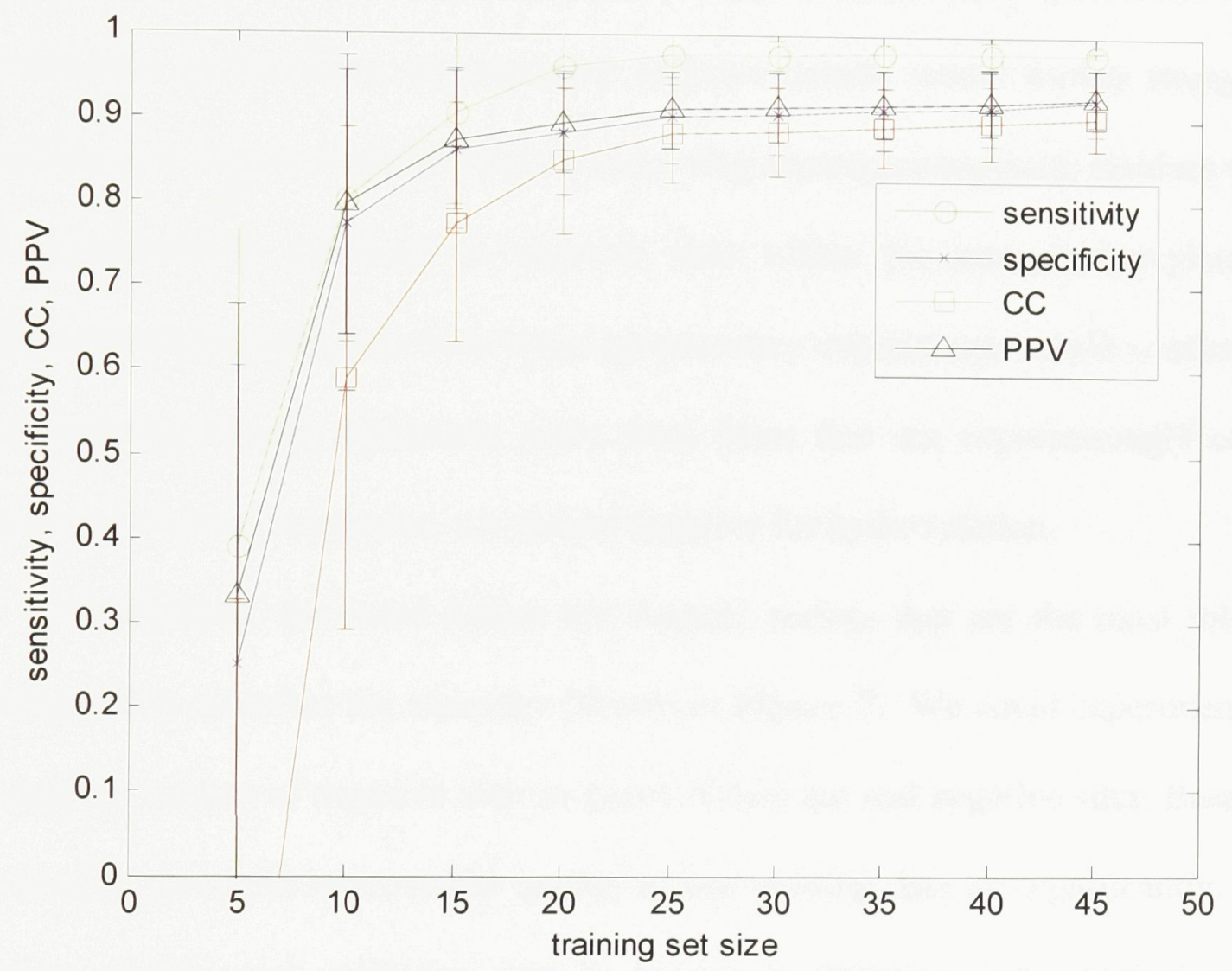


Our dataset of 61 positive points far exceeds this number, but more positive samples may still improve classification performance.

Second, our positive/negative class data were highly imbalanced. Figures 12, 13, 14, 15, 16, 17 and 18 showed the effect of this class imbalance. Even though the LIBSVM tool provides a parameter to adjust the misclassification cost ratio of positive/negative points during training, and optimization of this parameter improved classification performance, an improved negative dataset would further strengthen the classifier. The current study utilized all remaining asparagine/aspartate residues that were non-experimentally validated as negative sites within the same hydroxylated target protein. Mining spectra data from mass spectrometry experiments would confirm that all other asparagines and aspartates, aside from those that are experimentally confirmed hydroxylation sites in proteins, are indeed negative for hydroxylation.

In addition, we could utilize the support vectors that are the most informative training points to define the classifier (shown in Figure 7). We could experimentally test the support vectors of negative sites to check if they are real negative sites. Based on the test results, we could improve the quality of our training data set significantly. Iterative training, testing, and validating data is known as 'learning with queries' or 'active learning' (Duda et al., 2001)

\subsubsection{Compute different feature data}

In our pattern classification, we used the information from three different features, surface accessibility, secondary structure and PSSM, to train the classifier. Other similar 
studies (Julenius et al., 2004; Plewczynski et al., 2005) suggest using other features, such as sparse encoding, profile encoding, PSI-BLAST encoding, amino acid composition, etc. Therefore, other feature combinations could be tested for improved training.

\subsubsection{Effect of domain}

Within our 40 target proteins, many contained EGF and ankyrin domains. Proteins containing these domains were separated from ones that did not and the classifier trained on two datasets. Despite the separation of known domain proteins from datasets, the accuracy of the classifier was not improved. This may be due to the limited training data available, where any partitioning of the data results in insufficient training data. Therefore, with the future discovery of more proteins targeted by these hydroxylases added to datasets, those with known domains may affect the classifier in the future.

\subsubsection{Size of local sequence window}

Typically, these types of studies utilize a local sequence window size of 13 to 17 amino acids to perform the pattern searches (Mount, 2004). In the present study, a window size of 15 amino acids was chosen as a starting point for the reasons provided above. The question remains if 15 amino acids is the best sequence window size to use (as opposed to 13 or 17) to develop a hydroxylation site recognition and prediction system. To test this, I used WebLogo (Crooks et al., 2004) to align the sequence window 
of size 17 amino acids for the 61 positive sites. The conservation pattern is shown in Figure 22. A number of conclusions arise from this result; a) site 1 is mostly $\mathrm{C}$ or $\mathrm{L}, \mathrm{b}$ ) site 5 is primarily $\mathrm{C}, \mathrm{c}$ ) site 7 is mostly $\mathrm{C}$ or $\mathrm{D}$ and d) site 16 is primarily $\mathrm{C}$. These sites contain strong conservation patterns.

Various local sequence window sizes can be analyzed using Figure 22 as follows: a) 17 amino acids from sites 1 to 17 , b) 15 amino acids from sites 2 to 16 and c) 13 amino acids from sites 3 to 15 . It is clear that a sequence window of 13 amino acids will result in the loss of conservation information from sites 1 and 16. A sequence window of size 15 amino acids lost the conservation information of site 1. Figure 22 suggests that increasing the local sequence window size to 17 amino acids may provide useful data to the SVM, however one is cautioned to avoid increasing the dimensionality of the training data since it may result in reduced classification accuracy (Duda et al., 2001; Weiss and Kulikowski, 1991). This will be investigated in future work.

\subsubsection{Train classifiers for asparagine and aspartate hydroxylation separately}

We trained our classifier for asparagine/aspartate hydroxylation. The training data was the combination of $\mathrm{N}$ and $\mathrm{D}$. We want to know whether it would be better, if we train the classifier for $\mathrm{N}$ and $\mathrm{D}$ separately. To find out, I used WebLogo to align the sequence window of size 17 amino acids for the 44 positive $N$ sites and 17 positive $D$ sites separately as shown in Figure $\mathbf{2 3}$ and Figure 24, to see if they have their own distinct conservation pattern. 
Figure 22. The conservation pattern of sequence windows for the 61 positive sites from Sequence Logo. This Figure differs from Figure 10 in that a window of 17 residues is used rather than 15. The sequence window size is 17 as shown in X-axis. The 61 sequence windows aligned to show the pattern. The Y-axis represents the frequency of the amino acid presented at the window site. The bigger the letter, the more frequent the amino acid presented. It shows some conserved patterns, such as a) site 1 is mostly $\mathrm{C}$ or L, b) site 5 is primarily $\mathrm{G}, \mathrm{c}$ ) site 7 is mostly $\mathrm{C}$ or D and d) site 16 is primarily C. These sites contain strong conservation patterns. 


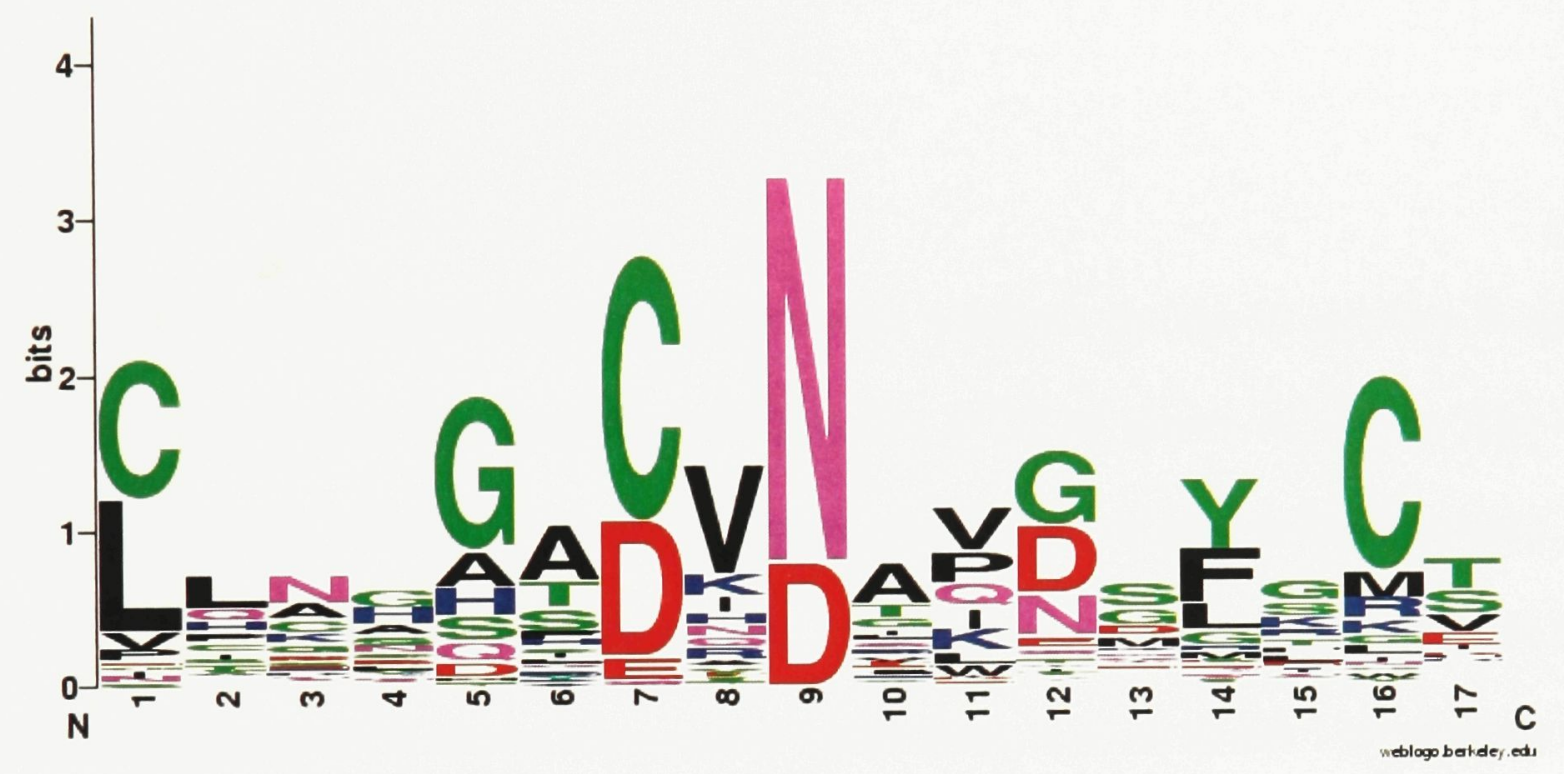


Figure 23. The conservation pattern of sequence windows for the 44 positive $\mathrm{N}$ sites from Sequence Logo. The sequence window size is 17 as shown in X-axis. The 44 sequence windows aligned to show the pattern. The Y-axis represents the frequency of the amino acid presented at the window site. The bigger the letter, the more frequent the amino acid presented. It shows some conserved patterns, such as a) site 1 is primarily L, b) site 5 is primarily $\mathrm{G}, \mathrm{c}$ ) site 7 is mostly $\mathrm{C}$ or $\mathrm{D}, \mathrm{d}$ ) site 12 is mostly $\mathrm{C}$ or $\mathrm{D}$ and e) site 16 is primarily $\mathrm{C}$. 


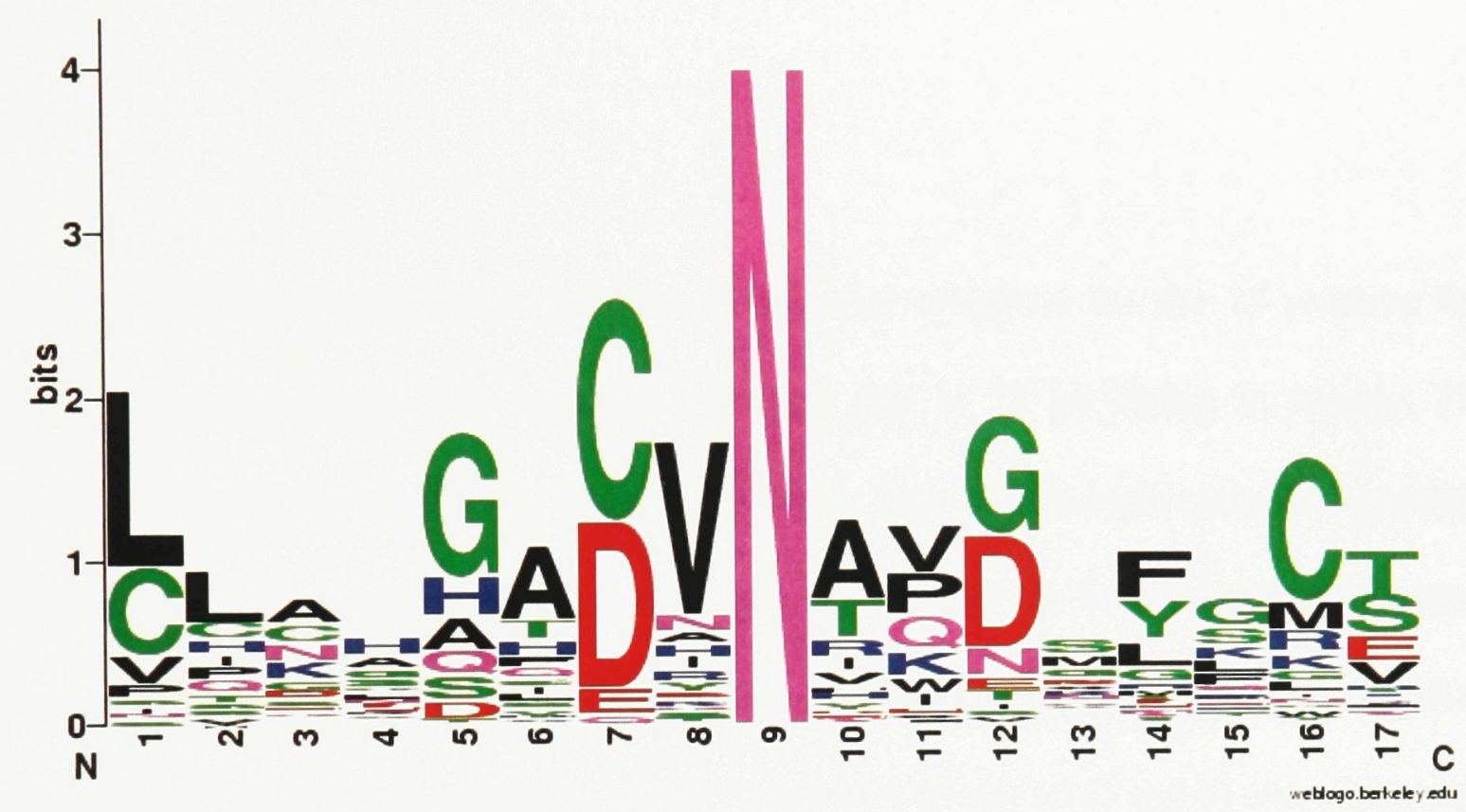


Figure 24. The conservation pattern of sequence windows for the 17 positive D sites from Sequence Logo. The sequence window size is 17 as shown in X-axis. The 17 sequence windows aligned to show the pattern. The Y-axis represents the frequency of the amino acid presented at the window site. The bigger the letter, the more frequent the amino acid presented. It shows very strong conserved patterns, such as a) site 1 is primarily $\mathrm{C}, \mathrm{b}$ ) site 5 is mostly $\mathrm{G}, \mathrm{c}$ ) site 7 and site 16 are all $\mathrm{C}$ and c) site 14 is mostly $\mathrm{Y}$ or F. 


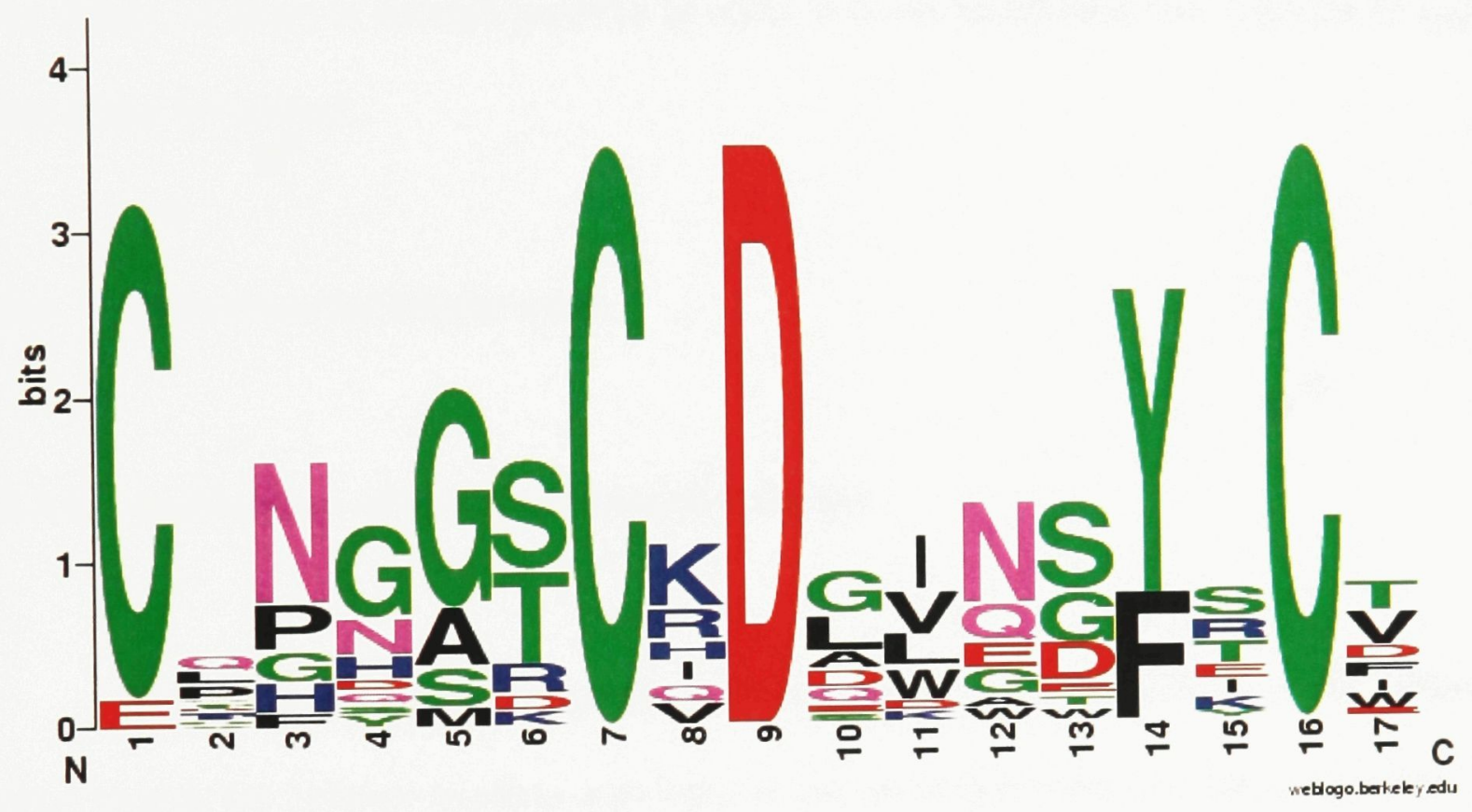


Comparing Figures 22, 23 and 24, the conservation pattern of $\mathrm{N}$ sites is similar to the conservation pattern of N/D sites, but the conservation pattern of D sites has its own distinct conservation pattern. For example, position 7 and position 16 are all C, and position 1 is primarily C. While this appears to be evidence of strong sequence conservation for D sites, it is based on only 17 positive data points, which is not enough to train the classifier as Figure 21 suggested. With the discovery of new protein targets in the future, if there is enough positive D sites, it is recommended that separate $\mathrm{N}$ and $\mathrm{D}$ classifiers be trained.

\subsection{Directions for future work}

\subsubsection{Application of tool to all human proteins}

Since the asparagine/aspartate hydroxylation is very important to the potential drug targets for cellular hypoxia survival, we are most interested in human proteins. We are currently predicting the asparagine/aspartate hydroxylation sites for all human proteins and will soon make it available for public use. It will provide researchers a ranked list for experimental verification.

\subsubsection{Application of current approach to other types of hydroxylation}

Since our model of developing the asparagine/aspartate hydroxylation prediction tool was successful, we could use this model to develop the prediction tools for other 
types of hydroxylation, such as proline hydroxylation, lysine hydroxylation, etc. Also, the protein databases from other organisms could be combined with human or searched separately for similar sites of hydroxylation. Searching databases other than human would depend upon the conservation of function of hydroxylases throughout evolution and their role in other vertebrates as well as eukaryotes in general. 


\section{REFERENCES}

Ahmad, S., Groumiha, M.M. and Sarai, A. 2003. Real value prediction of solvent accessibility from amino acid sequence. Proteins: Structure, Function, and Genetics. 50: 629-35.

Altschul, S.F., Madden, T.L., Schaffer, A.A., Zhang, J., Zhang, Z., Miller, W. and Lipman, D.J. 1997. Gapped BLAST and PSI-BLAST: a new generation of protein database search programs. Nucleic Acids Res. 25(17): 3389-402.

Bhagwat, M. and Aravind, L. 2007. PSI-BLAST tutorial. Methods Mol Biol. 395: 177-86.

Boser, B.E., Guyon, I.M. and Vapnik, V.N. 1992. A training algorithm for optimal margin classifiers. In: Haussler D, ed. $5^{\text {th }}$ Annual ACM Workshop on COLT. Pittsburgh (Pennsylvanian): ACM Press, 144-52.

Chang, C.C. and Lin, C.J. 2001. LIBSVM: a library for support vector machines. Software available at http://www.csie.ntu.edu.tw/ cjlin/libsvm. Accessed on 2 Sept. 2008.

Coleman, M.L. McDonough, M.A. Hewitson, K.S., Coles, C., Mecinovic, J., Edelmann, M. Cook, K.M., Cockman, M.E., Lancaster, D.E., Kessler, B.M., Oldham, N.J., Ratcliffe, P.J. and Schofield, C.J. 2007. Asparaginyl hydroxylation of the Notch ankyrin repeat domain by factor inhibiting hypoxia-inducible factor. J Biol Chem. 282(33): 24027-38.

Crooks, G.E., Hon, G., Chandonia, J.M. and Brenner, S.E. 2004. WebLogo: a sequence logo generator. Genome Res. 14(6): 1188-90. 
Duda, R.O., Hart, P.E. and Stork, D.G. 2001. Pattern classification. $2^{\text {nd }}$ edition. WileyInterscience.

Green, J.R., Dmochowski, G.M. and Golshani, A. 2006. Prediction of protein sumoylation sites via parallel cascade identification. CMBEC06, Vancouver.

Green, J.R., Korenberg, M.J. and Aboul-Magd, M.O. 2009. PCI-SS: MISO dynamic nonlinear protein secondary structure prediction. BMC Bioinformatics. 10: 222.

Gronke, R.S., VanDusen, W.J., Gardsky, V.M., Jacobs, J.W., Sardana, M.K., Stern, A.M. and Friedman, P.A. 1989. Aspartyl beta-hydroxylase: In vitro hydroxylation of a synthetic peptide based on the structure of the first growth factor-like domain of human factor IX. Proc. Natl. Acad. Sci. U.S.A. 86(10): 3609-13.

Henikoff, S. and Henikoff, J.G. 1992. Amino Acid substitution matrices from protein blocks. Proc. Natl. Acad. Sci. U.S.A. 89(22): 10915-9.

Hill, T. and Lewicki, P. 2007. Statistics: methods and applications. StatSoft Inc.

Hsu, C.W., Chang, C.C. and Lin, C.J. 2008. A practical guide to support vector classification. Department of Computer Science. National Taiwan University.

Jensen, O.N. 2008. Analysis of post-translational modifications by mass spectrometry. University of Southern Denmark, Amsterdam, NL. HUPO conference. Avalilable from http://www.hupo.org/educational/past_congresses/2008_Amsterdam/3.\%20HUP O_educational_Jensen.pdf. Accessed on 7 Feb. 2008.

Julenius, K., Molgaard, A., Gupta, R. and Brunak, S. 2004. Prediction, conservation analysis, and structural characterization of mammalian mucin-type Oglycosylation sites. Glycobiology. 15: 153-64. 
Lee, T.Y., Huang, H.D., Hung, J.H., Huang, H.Y., Yang, Y.S. and Wang, T.H. 2006. dbPTM: an information repository of protein post-translatinal modification. Nucleic Acids Res. 34 (Database issue): D622-7.

Mount, D.W. 2004. Sequence and genome analysis. $2^{\text {nd }}$ ed. Cold Spring Harbor, NY: Cold Spring Harbor Laboratory Press. 692: 456.

Plewczynski, D., Tkacz, A., Wyrwicz, L.S. and Rychlewski, L. 2005. AutoMotif server: prediction of single residue post-translational modifications in proteins. Bioinformatics. 21(10): 2525-7.

Przysiecki, C.T., Staggers, J.E., Ramjit, H.G., Musson, D.G., Stern, A.M., Bennett, C.D. and Friedman, P.A. 1987. Occurrence of beta-hydroxylated asparagin residues in non-vitamin K-dependent proteins containing epidermal growth factor-like domains. Proc Natl Acad Sci U.S.A. 84(22): 7856-60.

Sales, A.P., Tomara, G.D. and Kepler, T.B. 2008. Improving peptide-MHC class I binding prediction for unbalanced datasets. BMC Bioinformatics. 9: 385.

Sbodio, J.I. and Chi, N.W. 2002. Identification of a tankyrase-binding motif shared by IRAP, TAB182, and human TRF1 but not mouse TRF1. NuMA contains this RXXPDG motif and is a novel tankyrase partner. J Biol Chem. 277(35): 31887-92.

Scholkopf, A. and Smola, A. 2002. Learning with kernels. Cambridge (Massachusetts): MIT Press.

Stenflo, J., Lundwall, A. and Dahlback B. 1987. Beta-hydroxyasparagine in domains homologous to the epidermal growth factor precursor in vitamin $\mathrm{K}$-dependent protein S. Proc Natl Acad Sci U.S.A. 84(2): 368-72.

Vapnik, V. 1999. The nauture of statistical learning theory. $2^{\text {nd }}$ edition. Springer. 
Weiss, S.M. and Kulikowski, C.A.1991. Computer systems that learn: classification and prediction methods from statistics, neural nets, machine learning, and expert systems. Morgan Kaufmann Publishers Inc., San Francisco, CA.

Wikipedia contributors. 2009. Support vector machine. Wikipedia, The Free Encyclopedia. http://en.wikipedia.org/wiki/Support_vector_machine. (accessed September 2, 2009). 